This is the final version of:

Baldi, A., \& Franchi, B. (2015). Maxwell's equations in anisotropic media and carnot groups as variational limits. Advanced Nonlinear Studies, 15(2), 333-362. doi:

The final published version is available online at: http://dx.doi.org/10.1515/ans2015-0204

Rights / License:

The terms and conditions for the reuse of this version of the manuscript are specified in the publishing policy. For all terms of use and more information see the publisher's website.

This item was downloaded from IRIS Università di Bologna (https://cris.unibo.it/)

When citing, please refer to the published version. 


\title{
Maxwell's Equations in Anisotropic Media and Carnot Groups as Variational Limits
}

\author{
Annalisa Baldi, Bruno Franchi* \\ Dipartimento di Matematica \\ University of Bologna, 40126 Bologna, Italy \\ e-mail:annalisa.baldi2@unibo.it, bruno.franchi@unibo.it \\ Received 09 April 2014 \\ Communicated by Guozhen Lu
}

\begin{abstract}
Let $\mathbb{G}$ be a free Carnot group (i.e. a connected simply connected nilpotent stratified free Lie group) of step 2. In this paper, we prove that the variational functional generated by "intrinsic" Maxwell's equations in $\mathbb{G}$ is the $\Gamma$-limit of a sequence of classical (i.e. Euclidean) variational functionals associated with strongly anisotropic dielectric permittivity and magnetic permeability in the Euclidean space.
\end{abstract}

1991 Mathematics Subject Classification. 35Q61, 35R03, 58A10, 49J45.

Key words. Carnot groups, differential forms, Maxwell'equations, $\Gamma$-convergence

\section{Introduction}

Classical Maxwell's equations in the vacuum (neither charges nor currents) in $\mathbb{R}_{s} \times \mathbb{R}_{x}^{3}$ are

$$
\begin{array}{cc}
\frac{\partial \vec{B}}{\partial s}=-\operatorname{curl} \vec{E} \quad, \quad \operatorname{div} \vec{H}=0, \\
\frac{\partial \vec{E}}{\partial s}=\operatorname{curl} \vec{B} \quad, \quad \operatorname{div} \vec{E}=0,
\end{array}
$$

*The authors acknowledge the support of University of Bologna, funds for selected research topics, and the Gruppo Nazionale per l'Analisi Matematica, la Probabilità e le loro Applicazioni (GNAMPA) of the Istituto Nazionale di Alta Matematica (INdAM). 
where $\vec{E}$ and $\vec{B}$ are the electric and magnetic induction fields, respectively (see, e.g. [7], Section1.1).

Denote now by $\left(\Omega^{*}, d\right)$ the de Rham's complex of differential forms in $\mathbb{R}^{3}$ associated with the exterior differential $d$. If $\delta$ is the formal adjoint in $L^{2}$ of $d$, and $*$ is the standard Hodge-star operator in $\mathbb{R}^{3}$, then equations (1.1) and (1.2) can be reformulated in a simpler form as follows: we fix the standard volume form $d V$ in $\mathbb{R}^{3}$, and we consider a 2-form $F \in \Omega^{2}$ (Faraday's form), that can be always written as $F=d s \wedge E+B$, where $E$ is the electric field 1-form and $B$ is the magnetic induction 2-form. Relatioship between $E, B$ and the corresponding vector fields $\vec{E}, \vec{B}$ will be illustrated below. Then, classical Maxwell's equations become

$$
d F=0 \quad \text { and } \quad d\left(*_{M} F\right)=0
$$

Here $*_{M}$ is the Hodge-star operator associated with the space-time Minkowskian metric and the volume form $d s \wedge d V$ in $\mathbb{R} \times \mathbb{R}^{3}$.

Clearly, equations (1.3) make perfectly good sense in $\mathbb{R}^{n}$ with $n \geq 3$ (for physical meaning of Maxwell's equations in higher dimensions, we refer for instance to [34], Chapter 3).

Recently, in a series of papers ([5], [18], [19]), the authors introduced the notion of "intrinsic" Maxwell's equations in Carnot groups.

We recall that a connected and simply connected Lie group (G,.) (in general noncommutative) is said a Carnot group of step $\kappa$ if its Lie algebra $g$ admits a step $\kappa$ stratification, i.e. there exist linear subspaces $V_{1}, \ldots, V_{K}$ such that

$$
\mathfrak{g}=V_{1} \oplus \ldots \oplus V_{\kappa}, \quad\left[V_{1}, V_{i}\right]=V_{i+1}, \quad V_{\kappa} \neq\{0\}, \quad V_{i}=\{0\} \text { if } i>\kappa,
$$

where $\left[V_{1}, V_{i}\right]$ is the subspace of $\mathfrak{g}$ generated by the commutators $[X, Y]$ with $X \in V_{1}$ and $Y \in V_{i}$.

The first layer $V_{1}$, the so-called horizontal layer, plays a key role in the theory, since it generates the whole of $\mathfrak{g}$ by commutation.

The Carnot group $\mathbb{G}$ is said to be free if its Lie algebra is free, i.e, if the commutators satisfy no linear relations other than antisymmetry and Jacobi identity. Our main result will be proved in the setting of free Carnot groups.

A Carnot group $\mathbb{G}$ can be always identified, through exponential coordinates, with the Euclidean space $\mathbb{R}^{n}$, where $n$ is the dimension of $\mathfrak{g}$, endowed with a suitable group operation. The stratification of the Lie algebra induces a family of anisotropic dilations $\delta_{\lambda}$ $(\lambda>0)$ on $\mathfrak{g}$ and therefore, through exponential map, on $\mathbb{G}$.

In Section 2 we recall some basic results of the theory of Carnot groups. We refer to [14] or [6] for an detailed introduction.

From now on, we use the word "intrinsic" for notions in $\mathbb{G}$ depending only on the structure of its Lie algebra $\mathfrak{g}$.

The definition of Maxwell's equation in Carnot group is written in terms of differential forms and relies on the Rumin's theory of differential forms in Carnot groups ([29], [32], [30]). We refer to the Appendix for a description of Rumin's complex, as well as to [3]. Roughly speaking, Rumin defines a kind of "minimal" class $E_{0}^{*}$ of intrinsic forms as well as an exterior intrinsic differential $d_{c}$ that makes $\left(E_{0}^{*}, d_{c}\right)$ homotopic to the De Rham's complex. A crucial property of $d_{c}$, that will affect all the subsequent theory, is that, in general, $d_{c}$ is a differential operator in the horizontal derivatives of order greater than 1 . 
The complex $\left(E_{0}^{*}, d_{c}\right)$ being given, it is easy to define formally a set of intrinsic Maxwell's equations in a Carnot group $\mathbb{G}=\mathbb{R}^{n}$ as follows: we refer to [19] for the definition. By Theorem 5.5 in [19], Maxwell's equations in a $\mathbb{G}$ take the form

$$
\begin{gathered}
\frac{\partial(* B)}{\partial s}=* d_{c} E \quad, \quad \delta_{c}(* B)=0, \\
\frac{\partial E}{\partial s}=(-1)^{n} * d_{c}(* B) \quad, \quad \delta_{c} E=0,
\end{gathered}
$$

where $E \in E_{0}^{1}, B \in E_{0}^{2}$. Moreover $\delta_{c}$ and $*$ are associated with a suitable left-invariant scalar product. Notice that intrinsic Maxwell's equations are no more of order 1, depending on the order of $d_{c}$.

It has been shown in [19] that equations (1.4) and (1.5) are invariant under the action of a suitable class of contact Lorentz transformations (contact maps are algebra endomorphism that preserve the stratification).

In spite of the number of various applications of Carnot groups to describe different phenomena in applications, here we are not looking for any application modeling physical situations. In fact, Maxwell's equations in Carnot groups have been introduced in order to carry on the investigation of peculiar features of the geometry of the groups. Nevertheless, we want to mention that in the first Heisenberg group $\mathbb{H}^{1}$ equations (1.4) and (1.5) arise in the study of quasiconformal or quasiregular maps in $\mathbb{H}^{1}$, precisely as classical Maxwell's equations appear in quasiconformal map theory in the Euclidean setting (see [23], [1]).

The goal of the present paper is to show that intrinsic Maxwell's equation can be seen as (variational) limits of Riemannian Maxwell's equations in anisotropic media, in the spirit of the Gromov's Riemannian approximation. Riemannian approximations have been considered in different settings, among others, by Rumin [31], Ge [20], Citti \& Manfredini [8]. When dealing with Maxwell's equations, this approximation is obtained via classical Riemannian Maxwell's equations in the matter associated with real materials with strongly anisotropic dielectric permittivity and magnetic permeability.

To this end, we state preliminarily classical Maxwell equations in matter for timeharmonic vector fields. In bounded regions of the space, the study of Maxwell's equations, together with (say) relative boundary conditions, can be reduced to the study of stationary points of variational functionals ([9], [25], [24]). In some sense, these functionals contain all the structure of Maxwell's equations. In this paper, we prove precisely that the variational functional in the group generated by intrinsic Maxwell's equations is the $\Gamma$-limit of a sequence of the variational functionals associated with Euclidean strongly anisotropic dielectric permittivity and magnetic permeability. We notice that the correct formulation of the relative boundary condition in the group is not straightforward and relies on a new Green formula (see Theorem 4.2 below). In fact, the $\Gamma$-limit result depends strongly on the right choice of the boundary conditions.

We want to state explicitly that the $\Gamma$-convergence is not meant to derive existence results of solutions of the group Maxwell's equation, because of the lack of several wellknown crucial property in the limit functional, like coerciveness (think for instance of Gaffney's inequality) and higher order regularity properties of the stationary points. Instead, it is meant to show that the "structure" of the intrinsic system (1.4), (1.5) is the limit of the corresponding structure of a sequence of "true" equations in the matter. 
When we try to compare Maxwell's equation in G with usual Maxwell's equations in the Euclidean space $\mathbb{R}^{n}$, different scalar products are involved. Thus we must proceed carefully and establish precise definitions to take into account the two different (superposed) structures. Indeed, all our equations are written on the exterior algebra of the cotangent bundle of $\mathbb{G}$, but they are associated with different scalar products, coming ultimately, from two different group structures on $\mathbb{G}$, i.e. $(\mathbb{G}, \cdot)$ and the Abelian Euclidean group $(\mathbb{G},+)$.

The paper is organized as follows: In Section 2 we recall some basic facts about Carnot groups, but most of the property and definitions about Carnot groups are contained in the Appendix. In Section 3, we write Maxwell's equations in the matter in the Euclidean space $\mathbb{R}^{n}$. Starting from Section 4, we suppose $\mathbb{G}$ to be a free Carnot group of step two. We recall the notion of "intrinsic" Maxwell's equations and we relate them to an intrinsic variational functional. Section 5 contains our main results. In particular we prove that if $\mathbb{G}$ is a free Carnot group of step two, then the variational functional in the group, generated by intrinsic Maxwell's equations, is a $\Gamma$-limit of a class of variational functional in $\mathbb{R}^{n}$ with strongly anistropic dielectric permittivity and magnetic permeability. Finally, in Section 6, we provide some explicit computations in the first Heisenberg group $\mathbb{H}^{1}$.

To keep the paper self-contained, we add an appendix that contains some basic notions and results concerning Carnot group, as well as a self-contained sketch of Rumin's theory.

\section{Notations and preliminary results: two scalar products}

Let $(\mathbb{G}, \cdot)$ be a Carnot group of step $\kappa$ identified to $\mathbb{R}^{n}$ through exponential coordinates, as defined in the Introduction.

As customary, we denote by $T \mathbb{G}$ the tangent fiber bundle of $\mathbb{G}$, and by $T \mathbb{G}_{x}$ its fiber over $x \in \mathbb{G}$.

By definition, $\mathfrak{g}$, the Lie algebra of $\mathbb{G}$ (i.e. the Lie algebra of the left-invariant vector fields on $\mathbb{G}$ ) can be written as

$$
\mathfrak{g}=V_{1} \oplus \ldots \oplus V_{\kappa}, \quad\left[V_{1}, V_{i}\right]=V_{i+1}, \quad V_{\kappa} \neq\{0\}, \quad V_{i}=\{0\} \text { if } i>\kappa .
$$

Set $m_{i}=\operatorname{dim}\left(V_{i}\right)$, for $i=1, \ldots, \kappa$ and $h_{i}=m_{1}+\cdots+m_{i}$ with $h_{0}=0$. Clearly, $h_{\kappa}=n$. We denote by $Q$ the homogeneous dimension of $\mathbb{G}$, i.e. we set

$$
Q:=\sum_{i=1}^{\kappa} i \operatorname{dim}\left(V_{i}\right) .
$$

If $e$ is the unit element of $(\mathbb{G}, \cdot)$, we recall that the map $X \rightarrow X(e)$, that associate with a left-invariant vector field $X$ its value at $e$, is an isomorphism from $\mathfrak{g}$ to $T \mathbb{G}_{e}$, in turn identified with $\mathbb{R}^{n}$. From now on, we shall use systematically these identifications.

We choose now a basis $e_{1}, \ldots, e_{n}$ of $\mathbb{R}^{n}$ adapted to the stratification of $\mathfrak{g}$, i.e. such that

$$
e_{h_{j-1}+1}, \ldots, e_{h_{j}} \text { is a basis of } V_{j} \text { for each } j=1, \ldots, \kappa \text {. }
$$

Then, we denote by $\langle\cdot, \cdot\rangle$ the scalar product in $g$ making the adapted basis $\left\{e_{1}, \ldots, e_{n}\right\}$ orthonormal. Moreover, let $X=\left\{X_{1}, \ldots, X_{n}\right\}$ be the family of left invariant vector fields such that $X_{i}(e)=e_{i}, i=1, \ldots, n$. Clearly, $X$ is orthonormal with respect to $\langle\cdot, \cdot\rangle$. 
The dual space of $\mathfrak{g}$ is denoted by $\bigwedge^{1} \mathfrak{g}$. The basis of $\bigwedge^{1} \mathfrak{g}$, dual of the basis $X_{1}, \cdots, X_{n}$, is the family of covectors $\left\{\theta_{1}, \cdots, \theta_{n}\right\}$. We indicate by $\langle\cdot, \cdot\rangle$ also the inner product in $\bigwedge^{1} \mathfrak{g}$ that makes $\theta_{1}, \cdots, \theta_{n}$ an orthonormal basis. We point out that, except for the trivial case of the commutative group $\mathbb{R}^{n}$, the forms $\theta_{1}, \cdots, \theta_{n}$ may have polynomial (hence variable) coefficients.

Following Federer (see [12] 1.3), the exterior algebras of $\mathfrak{g}$ and of $\Lambda^{1} \mathfrak{g}$ are the graded algebras indicated as $\bigwedge_{*} \mathfrak{g}=\bigoplus_{h=0}^{n} \bigwedge_{h} \mathfrak{g}$ and $\bigwedge^{*} \mathfrak{g}=\bigoplus_{h=0}^{n} \bigwedge^{h} \mathfrak{g}$ where $\Lambda_{0} \mathfrak{g}=\Lambda^{0} \mathfrak{g}=\mathbb{R}$ and, for $1 \leq h \leq n$,

$$
\begin{aligned}
& \bigwedge_{h} \mathfrak{g}:=\operatorname{span}\left\{X_{i_{1}} \wedge \cdots \wedge X_{i_{h}}: 1 \leq i_{1}<\cdots<i_{h} \leq n\right\}, \\
& \bigwedge^{h} \mathfrak{g}:=\operatorname{span}\left\{\theta_{i_{1}} \wedge \cdots \wedge \theta_{i_{h}}: 1 \leq i_{1}<\cdots<i_{h} \leq n\right\} .
\end{aligned}
$$

The elements of $\bigwedge_{h} \mathfrak{g}$ and $\bigwedge^{h} \mathfrak{g}$ are called $h$-vectors and $h$-covectors. The dual space $\bigwedge^{1}\left(\bigwedge_{h} \mathfrak{g}\right)$ of $\bigwedge_{h} \mathfrak{g}$ can be naturally identified with $\bigwedge^{h} \mathfrak{g}$.

Definition 2.1 We denote by $\Theta^{h}$ the basis $\left\{\theta_{i_{1}} \wedge \cdots \wedge \theta_{i_{h}}: 1 \leq i_{1}<\cdots<i_{h} \leq n\right\}$ of $\wedge^{h} \mathfrak{g}$. The inner product $\langle\cdot, \cdot\rangle$ extends canonically to $\bigwedge_{h} \mathfrak{g}$ and to $\wedge^{h} \mathfrak{g}$ making the bases $X_{i_{1}} \wedge \cdots \wedge X_{i_{h}}$ and $\theta_{i_{1}} \wedge \cdots \wedge \theta_{i_{h}}$ orthonormal.

We can define now, as usual, two families of vector bundles, denoted by $\bigwedge_{*}(T \mathbb{G})$ (the exterior power of the tangent bundle) and $\bigwedge^{*}(T \mathbb{G}$ ) (the exterior power of the cotangent bundle) over $\mathbb{G}$.

Sections of $\bigwedge^{h}(T \mathbb{G})$ are differential $h$-forms, and sections of $\bigwedge_{h}(T \mathbb{G})$ are $h$-vector fields, $1 \leq h \leq n$. If $\mathcal{U} \subset \mathbb{G}$ is an open set, we denote by $\Omega^{h}(\mathcal{U})$ and $\Omega_{h}(\mathcal{U})$ the spaces of $h$-forms and of $h$-vectors in $\mathcal{U}$. If $\mathcal{U}=\mathbb{G}$, we write simply $\Omega^{h}$ and $\Omega_{h}$.

With the notations of [21], Chapter 2, Section 2.1, if $V, W$ are finite dimensional linear vector spaces and $L: V \rightarrow W$ is a linear map, we denote by

$$
\Lambda^{h} L: \bigwedge^{h} W \rightarrow \bigwedge^{h} V
$$

the linear map induced by $L$ between exterior powers (see [21], Chapter 2, Section 2.1).

We stress now that the aim of this paper is to compare Maxwell's equation in $\mathbb{G}$ with usual Maxwell's equations in the Euclidean space $\mathbb{R}^{n}$. Since different scalar products are involved, we must proceed carefully and to establish precise definitions to take into account the two different (superposed) structures. Indeed, all our equations are written on the exterior algebra of the cotangent bundle of $\mathbb{G}$, but they are associated with different scalar products, coming ultimately, from two different group structures on $\mathbb{G}$, i.e. $(\mathbb{G}, \cdot)$ and the Abelian Euclidean group $(\mathbb{G},+)$.

In particular for any $x \in \mathbb{G}$ two families of (left) translations $\tau_{x}: \mathbb{G} \rightarrow \mathbb{G}$ and $t_{x}: \mathbb{G} \rightarrow \mathbb{G}$ are defined respectively by

$$
\tau_{x} p:=x \cdot p, \quad t_{x} p:=x+p
$$


Thanks to the identification of $T \mathbb{G}_{e}$ with $\mathfrak{g}$, the maps

$$
(x, \alpha) \longrightarrow\left(x, \Lambda^{h}\left(d \tau_{x}(e)\right) \alpha\right) \text { from } \bigwedge^{h}(T \mathbb{G}) \text { to } \mathbb{G} \times \bigwedge^{h} \mathfrak{g}
$$

and

$$
(x, \alpha) \longrightarrow\left(x, \Lambda^{h}\left(d t_{x}(e)\right) \alpha\right) \text { from } \bigwedge^{h}(T \mathbb{G}) \text { to } \mathbb{G} \times \bigwedge^{h} \mathfrak{g}
$$

define two canonical (global) trivializations of the vector bundle $\wedge^{h}(T \mathbb{G})$ associated with the two families $\tau_{x}$ and $t_{x}$ of left translations in $\mathbb{G}$.

Starting from the scalar product defined in $\bigwedge^{h} \mathfrak{g}$, each trivialization defines on the fiber $\bigwedge^{h}(T \mathbb{G})_{p}$ over $p \in \mathbb{G}$ two scalar products

$$
\left\langle\Lambda^{h} d \tau_{p^{-1}}(\alpha), \Lambda^{h} d \tau_{p^{-1}}(\beta)\right\rangle_{p}:=\langle\alpha, \beta\rangle
$$

and

$$
\left\langle\Lambda^{h} d t_{-p}(\alpha), \Lambda^{h} d t_{-p}(\beta)\right\rangle_{p}:=\langle\alpha, \beta\rangle_{\text {Euc }}
$$

Analogously, $\tau$ and $t$ define on $\bigwedge^{h}(T \mathbb{G})_{p}$ two (orthonormal) bases $\Theta_{p}^{h}$ and $\Theta_{p}^{h \text {,Euc }}$ through the identities

$$
\Theta_{p}^{h}:=\Lambda^{h} d \tau_{p^{-1}}(e) \Theta^{h} \quad \text { and } \quad \Theta_{p}^{h, \mathrm{Euc}}:=\Lambda^{h} d t_{-p}(e) \Theta^{h} .
$$

In particular,

$$
\Theta_{p}^{h, \text { Euc }}=\Lambda^{h}\left(d t_{-p}(p)\right) \Lambda^{h}\left(d \tau_{p}(e)\right) \Theta_{p}^{h} .
$$

Obviously, a parallel argument can be carried out for $h$-vectors in $\bigwedge_{h}(T \mathbb{G})$.

From now on, if $\alpha \in \bigwedge^{h} \mathfrak{g}$, we denote by $\alpha_{\mathfrak{E}}=\left(\alpha_{\mathfrak{E}, 1}, \alpha_{\mathfrak{E}, 2,2}, \ldots\right)$ and by $\alpha_{\mathfrak{g}}=\left(\alpha_{\mathfrak{g}, 1}, \alpha_{\mathfrak{g}, 2}, \ldots\right)$ the vector of its coordinates with respect to the bases $\Theta^{h, \text { Euc }}$ and $\Theta^{h}$, respectively.

It is easy to see that

$$
\langle\alpha, \beta\rangle=\alpha_{\mathfrak{g}} \bullet \beta_{\mathfrak{g}} \quad \text { and }\langle\alpha, \beta\rangle_{\mathrm{Euc}}=\alpha_{\mathfrak{E}} \bullet \beta_{\mathfrak{E}},
$$

where $\bullet$ denotes the Euclidean scalar product in $\mathbb{R}^{N}$, with $N=\left(\begin{array}{l}n \\ h\end{array}\right)$.

Definition 2.2 The dual space $\bigwedge^{1}\left(\bigwedge_{h} \mathfrak{g}\right)$ of $\bigwedge_{h} \mathfrak{g}$ can be naturally identified with $\bigwedge^{h} \mathfrak{g}$.

The action of a $h$-covector $\varphi$ on a $h$-vector $v$ is denoted as $\langle\varphi \mid v\rangle$. The same notation is used for the duality between forms and vector fields.

If $v \in \Omega_{h}$ we define $v^{\natural} \in \Omega^{h}$ by the identity

$$
\left\langle v^{\natural} \mid w\right\rangle:=\langle v, w\rangle, \quad\left\langle v^{\natural, \mathrm{Euc}} \mid w\right\rangle:=\langle v, w\rangle_{\mathrm{Euc}}
$$

and analogously we define $\varphi^{\natural} \in \Omega_{h}$ and $\varphi^{\natural \text {,Euc }} \in \Omega_{h}$ for $\varphi \in \Omega^{h}$.

However, to avoid cumbersome notations, in the sequel we write also $v^{\natural}$ and $\phi^{\natural}$ with respect to the Euclidean scalar product, if this is evident from the context. 


\section{Maxwell's equations in matter}

Classical Maxwell's equations in a bounded smooth connected region $\mathcal{U} \subset \mathbb{R}^{3}$, for timeharmonic vector fields in $\mathbb{R}_{s} \times \mathbb{R}_{x}^{3}$

$$
e^{i \omega s} \vec{E}, e^{i \omega s} \vec{H}, e^{i \omega s} \vec{B}, e^{i \omega s} \vec{D},
$$

with no charges and no currents, read as follows:

$$
\begin{gathered}
\operatorname{curl} \vec{H}-i \omega \vec{D}=0 \quad \text { in } \mathcal{U}, \\
\operatorname{curl} \vec{E}+i \omega \vec{B}=0 \quad \text { in } \mathcal{U}, \\
\operatorname{div} \vec{D}=0, \quad \operatorname{div} \vec{B}=0 \quad \text { in } \mathcal{U},
\end{gathered}
$$

together with the constitutive relations

$$
\vec{D}=\varepsilon \vec{E}, \quad \vec{B}=\mu \vec{H},
$$

and the boundary condition

$$
\vec{E}_{\tan _{\partial \mathcal{U}}}=\vec{F} \quad \text { on } \partial \mathcal{U}
$$

Here $\varepsilon=\varepsilon(x), \mu=\mu(x) \in G L\left(\mathbb{R}^{3}\right)$ are invertible linear maps depending (say) smoothly on $x$ and $\vec{F}$ is a tangent vector field on $\partial \mathcal{U}$. Usually, the matrices $[\varepsilon],[\mu]$ are called respectively the dielectric permittivity and the magnetic permeability.

From previous equations we get the following differential equation satisfied by $\vec{D}$.

$$
\operatorname{curl} \mu^{-1} \operatorname{curl} \varepsilon^{-1} \vec{D}=\omega^{2} \vec{D} \text {. }
$$

Consider now the 1-forms $E=\vec{E}^{\natural}, H=\vec{H}^{\natural}$ and the 1-forms $* D:=-\vec{D}^{\natural}$ and $* B:=-\vec{B}^{\natural}$. The duality in the previous expression is meant with respect to the Euclidean scalar product $\langle\cdot, \cdot\rangle_{\text {Euc }}$, as stated in Definition 2.2. Since, trough all this section, we shall refer only to the "Euclidean" duality between forms and vector fields, in the sequel, we shall write e.g. $D^{\natural}$ instead of $D^{\natural, \text { Euc }}$.

On the other hand, keeping in mind that, if $\vec{V}$ is a vector field in $\mathbb{R}^{3}$, then curl $\vec{V}=$ $\left(* d \vec{V}^{\natural}\right)^{\natural}$. From the equations above we obtain

$$
* d E=i \omega * B, \quad, \quad \delta * B=0,
$$

and

$$
* d H=-i \omega * D, \quad, \quad \delta * D=0,
$$

together with the constitutive relations

$$
* B=\left(\Lambda^{1} \mu^{*}\right) H, \quad * D=\left(\Lambda^{1} \varepsilon^{*}\right) E .
$$

If we replace equation (3.8) in equation (3.9), taking also into account the constitutive relations (3.10), we get

$$
* d\left(\Lambda^{1} \mu^{*}\right)^{-1} * d\left(\Lambda^{1} \varepsilon^{*}\right)^{-1} * D=\omega^{2} * D .
$$

Thus, we have the following result. 
Proposition 3.1 Let $\vec{D} \in \Omega_{1}(\mathcal{U})$ be a solution of the differential equation (3.7). Then the 1 -form $\alpha=* D \in \Omega^{1}(\mathcal{U})$ satisfies the differential equation

$$
\delta M d N \alpha-\omega^{2} \alpha=0, \quad \delta \alpha=0
$$

where $M:=(\operatorname{det}[\mu])^{-1}\left(\Lambda^{2} \mu\right), N:=\Lambda^{1}\left(\varepsilon^{*}\right)^{-1}$. Moreover the boundary condition (3.6) becomes

$$
t(N \alpha)=\phi,
$$

where $\phi$ is a 1-form on $\partial \mathcal{U}$ and $t(N \alpha)$ is the tangential part of $N \alpha$ (see [21], Section 2.6, and Proposition 4.3 below).

Proof. As shown above, the equation in (3.7) is equivalent to equation (3.11).

Since on a $h$-forms

$$
* d=(-1)^{h+1} \delta *,
$$

then

$$
\delta *\left(\Lambda^{1} \mu^{*}\right)^{-1} * d\left(\Lambda^{1} \varepsilon^{*}\right)^{-1} * D-\omega^{2} * D=0 .
$$

Moreover

$$
*\left(\Lambda^{1}\left(\mu^{*}\right)^{-1}\right) *=* *(\operatorname{det}[\mu])^{-1}\left(\Lambda^{2} \mu\right)=(\operatorname{det}[\mu])^{-1}\left(\Lambda^{2} \mu\right) .
$$

Hence, we get that $* D$ satisfies (3.12), and we are done.

Notice that, by $(3.12), * D$ is a stationary point of the functional

$$
J^{\mu, \varepsilon}(\alpha):=\int_{\mathcal{U}}\langle M d N \alpha, d N \alpha\rangle_{\mathrm{Euc}} d V-\omega^{2} \int_{\mathcal{U}}\langle N \alpha, \alpha\rangle_{\mathrm{Euc}} d V
$$

in $\left\{\alpha \in W^{1,2}\left(\mathcal{U}, \bigwedge^{1} T \mathbb{R}^{3}\right), t(N \alpha)=\phi\right.$ in $\left.\partial \mathcal{U}\right\}=: W_{D N}^{1,2}\left(\mathcal{U}, \bigwedge^{1} T \mathbb{R}^{3}\right)$.

A standard approach to the Dirichlet problem with relative boundary conditions in a bounded open set $\mathcal{U}$ for system (3.12) relies on a variational approach combined with a compactness argument (see, for instance, [9] and Appendix A of [24]) for the functional

$$
\begin{aligned}
\tilde{J}^{\mu, \varepsilon}(\alpha):= & \int_{\mathcal{U}}\langle M d N \alpha, d N \alpha\rangle_{\text {Euc }} d V+\sigma \int_{\mathcal{U}}|\delta \alpha|^{2} d V \\
& +C \int_{\mathcal{U}}\langle N \alpha, \alpha\rangle_{\text {Euc }} d V
\end{aligned}
$$

in $W_{D N}^{1,2}\left(\mathcal{U}, \wedge^{1} \mathbb{R}^{3}\right)$. Here, $\sigma>0$ is a positive parameter, and $C>0$ is a large constant.

We stress that all the peculiarities of the structure of Maxwell's equations are contained in the functional (3.14). Indeed, the further steps of the technique rely on standard compactness arguments and on regularity properties of solutions of elliptic pde's.

Finally, we notice that (3.12) and all the previous arguments make perfect sense in $\mathbb{R}^{n}$ for any $n \in \mathbb{N}$. 
Indeed, let $\mathcal{U} \subset \mathbb{R}^{n}$ be a smooth connected region. Let $\varepsilon=\varepsilon(x), \mu=\mu(x) \in G L\left(\mathbb{R}^{n}\right)$ be invertible linear maps depending (say) smoothly on $x$. Consider two differential forms $E$ and $H$ in $\mathcal{U}$, with $E \in \Omega^{1}(\mathcal{U})$ and $H \in \Omega^{n-2}(\mathcal{U})$. If $*$ denotes the Hodge-star operator in $\mathbb{R}^{n}$, the n-dimensional Maxwell's equations for time-harmonic forms in an anisotropic medium read as follows,

$$
* d E=i \omega\left(\Lambda^{n-2} \mu^{*}\right) H, \quad, \quad \delta\left(\Lambda^{n-2} \mu^{*}\right) H=0
$$

and

$$
* d H=(-1)^{n} i \omega\left(\Lambda^{1} \varepsilon^{*}\right) E, \quad, \quad \delta\left(\Lambda^{1} \varepsilon^{*}\right) E=0 .
$$

Arguing as above, the 1-form $\alpha=\left(\Lambda^{1} \varepsilon^{*}\right) E$ satisfies equation (3.12). Again, the study of equation (3.12) can be reduced to the study of a functional akin to (3.14).

\section{Maxwell's equations in group and related functionals}

From now on, we denote by $\left(E_{0}^{*}, d_{c}\right)$ the complex of intrinsic differential forms that is described in detail in the Appendix.

If we consider the time-harmonic differential forms $e^{i \omega s} E, e^{i \omega s} H$ in the "free" space $\mathbb{R}_{s} \times \mathbb{G}_{x}$, where $E \in E_{0}^{1}(\mathbb{G})$ and $H \in E_{0}^{n-2}(\mathbb{G})$, equations (1.4) and (1.5) became

$$
\begin{gathered}
* d_{c} E=i \omega H \quad * d_{c} H=-i \omega E, \\
\delta_{c} H=0 \quad \delta_{c} E=0 .
\end{gathered}
$$

Hence, $\alpha=E$ satisfy

$$
\delta_{c} d_{c} \alpha-\omega^{2} \alpha=0 \quad \delta_{c} \alpha=0 .
$$

Clearly, if we consider equations (4.15) and (4.16) in the open set $\mathcal{U}$, we must couple equation (4.17) with suitable boundary conditions (see below). Then $\alpha$ is also a stationary point of the functional

$$
\int_{\mathcal{U}}\left|d_{c} \alpha\right|^{2} d V-\omega^{2} \int_{\mathcal{U}}|\alpha|^{2} d V
$$

Mimicking the Euclidean approach we have to consider the functional

$$
\int_{\mathcal{U}}\left|d_{c} \alpha\right|^{2} d V+\sigma \int_{\mathcal{U}}\left|\delta_{c} \alpha\right|^{2} d V+C \int_{\mathcal{U}}|\alpha|^{2} d V
$$

which still hides all the peculiarities of the structure of our intrinsic Maxwell's equations.

So far, our argument are rather vague. To make it accurate, we need to set precisely the functions spaces involved in our problem, as well as the boundary condition and to prove a formula of integration by parts for $d_{c}$ and $\delta_{c}$ (see Theorem 4.2 below).

First of all, since from now on we focus our interest in the functionals instead of the equation, without loss of generality we take $\sigma=1$. The natural setting for the functional (4.18) is provided by Folland-Stein function spaces that are left-invariant and grouphomogeneous.

Since here we are dealing only with integer order spaces, we can give this simpler definition (for a general presentation, see e.g. [13]). 
Definition 4.1 Let $\mathcal{U} \subset \mathbb{G}$ be an open set. If $1<s<\infty$ and $m \in \mathbb{N}$, then the space $W_{\mathbb{G}}^{m, s}(\mathcal{U})$ is the space of all $u \in L^{s}(\mathcal{U})$ such that

$$
X^{I} u \in L^{s}(\mathcal{U}) \text { for all multi-index } I \text { with } d(I)=m,
$$

endowed with the natural norm.

We recall that

Proposition 4.1 Let $\mathcal{U} \subset \mathbb{G}$ be an open set. If $1<s<\infty$ and $m \in \mathbb{N}$, then the space $W_{\mathbb{G}}^{m, s}(\mathcal{U})$ is independent of the choice of $X_{1}, \ldots, X_{m_{1}}$.

Proposition 4.2 Let $\mathcal{U} \subset \mathbb{G}$ be an open set. If $1<s<\infty$ and $m \in \mathbb{N}$, then $\mathcal{E}(\mathcal{U}) \cap W_{\mathbb{G}}^{m, s}(\mathcal{U})$ is dense in $W_{\mathbb{G}}^{m, s}(\mathcal{U})$.

The following result summarizes several extension and trace results for Folland-Stein spaces in Carnot groups (see Theorems 10.4 and 13.5 in [11] and Theorem B in [26]). All these results can be applied thanks to the characterization of uniform domains given [27] (see also [15]).

Theorem 4.1 Suppose $\mathbb{G}$ is a step 2 Carnot group. Let $\mathcal{U} \subset \mathbb{G}$ be an open connected smooth region, locally lying on one side of its boundary $\partial \mathcal{U}$. Then

i) $\mathcal{E}(\overline{\mathcal{U}})$ is dense in $W_{\mathbb{G}}^{m, s}(\mathcal{U})$;

ii) there exists a bounded linear map

$$
\mathcal{P}: W_{\mathbb{G}}^{1,2}(\mathcal{U}) \longrightarrow W_{\mathbb{G}}^{1,2}(\mathbb{G}) \text { such that } \quad \mathcal{P} u_{\mid \mathcal{U}}=u
$$

for any $u \in W_{\mathbb{G}}^{1,2}(\mathcal{U}) ;$

iii) the linear map

$$
\gamma: \mathcal{E}(\overline{\mathcal{U}}) \longrightarrow \mathcal{E}(\partial \mathcal{U}), \quad \gamma u=u_{\mid \partial \mathcal{U}}
$$

can be continued as a bounded map

$$
\gamma: W_{\mathbb{G}}^{1,2}(\mathcal{U}) \longrightarrow L^{2}(\partial \mathcal{U}, d \sigma),
$$

where

$$
d \sigma=\left(\sum_{j=1}^{m}\left\langle X_{j}, v\right\rangle^{2}\right)^{1 / 2} d \mathcal{H}^{n-1},
$$

$v$ being the outward unit normal to $\partial \mathcal{U}$.

Definition 4.2 Let $\mathcal{U} \subset \mathbb{G}$ be an open set. If $0 \leq h \leq n, 1 \leq s \leq \infty$ and $m \geq 0$, we denote by $W_{G}^{m, s}\left(\mathcal{U}, \Lambda^{h} \mathfrak{g}\right)$ the space of all sections of $\bigwedge^{h} \mathfrak{g}$ such that their components with respect to the basis $\Theta^{h}$ belong to $W_{\mathbb{G}}^{m, s}(\mathcal{U})$, endowed with its natural norm. Clearly, this definition is independent of the choice of the basis itself.

Moreover, if $E_{0}^{h}$ is the class of the intrinsic $h$-forms, then the space $W_{\mathbb{G}}^{m, s}\left(\mathcal{U}, E_{0}^{h}\right)$ are defined analogously, replacing $\Theta^{h}$ by $\Xi^{h}$ (see Remark 7.2 in the Appendix).

Obviously, Propositions 4.1, 4.2 and Theorem 4.1 still hold for form-valued spaces. 
If $\alpha \in \mathbf{C}^{\infty}\left(\partial \mathcal{U}, \bigwedge^{h} \mathfrak{g}\right)$, as in [21], Section 2.6, we denote by $t(\alpha)$ and $n(\alpha)$ the tangential and the normal parts of $\alpha$, respectively. We stress that both $t$ and $n$ can be extended to continuous linear maps from $L^{2}\left(\partial \mathcal{U}, \bigwedge^{h} \mathfrak{g}\right)$ to $L^{2}\left(\partial \mathcal{U}, \bigwedge^{h} \mathfrak{g}\right)$, since for any $x \in \partial \mathcal{U}$ $|\alpha(x)|^{2}=|t(\alpha)(x)|^{2}+|n(\alpha)(x)|^{2}$. It is important to stress that here and in the sequel, the space $L^{2}\left(\partial \mathcal{U}, \bigwedge^{h} \mathrm{~g}\right)$ has to be understood with respect to the measure $d \sigma$.

Thus, the following proposition follows by Theorem 4.1.

Proposition 4.3 If $\alpha \in \mathbf{C}^{\infty}\left(\overline{\mathcal{U}}, \wedge^{h} \mathfrak{g}\right)$ and we denote by $\gamma$ the trace operator

$$
\gamma: \mathbf{C}^{\infty}\left(\overline{\mathcal{U}}, \bigwedge^{h} \mathfrak{g}\right) \rightarrow \mathbf{C}^{\infty}\left(\partial \mathcal{U}, \bigwedge^{h} \mathfrak{g}\right), \quad \gamma(\alpha)=\left.\alpha\right|_{\partial \mathcal{U}},
$$

the maps

$$
\alpha \longrightarrow t(\gamma(\alpha)), \quad \alpha \longrightarrow n(\gamma(\alpha))
$$

can be extended as linear continuous maps from $W_{\mathbb{G}}^{1,2}\left(\mathcal{U}, \wedge^{h} \mathfrak{g}\right)$ to $L^{2}\left(\partial \mathcal{U}, \wedge^{h} \mathfrak{g}\right)$.

For the sake of simplicity, from now on, if $\alpha \in W_{\mathbb{G}}^{1,2}\left(\mathcal{U}, \wedge^{h} \mathfrak{g}\right)$, we write $t(\alpha)$ and $n(\alpha)$ instead of $t(\gamma(\alpha))$ and $n(\gamma(\alpha))$, respectively. This notation, though usually established in the literature, may yield ambiguities in our setting. A point must be stressed: we apply first the trace operator, and then we decompose the trace in his tangential and normal parts. In fact, it might be possible to decompose first the form in a neighborhood of the boundary, and to apply later the trace operator. This distinction would not have any influence in the Euclidean setting, but here is crucial. Indeed the decomposition is not compatible with the Folland-Sobolev spaces, since it mixes variable of different layers of the stratification.

Assumption. From now on, we assume $\mathbb{G}$ is a free Carnot group of step 2.

We recall that, roughly speaking, the group $\mathbb{G}$ is said to be free if its Lie algebra is free, i.e. the commutators satisfy no linear relationships other than antisymmetry and the Jacobi identity. This is a large and relevant class of Carnot groups. We recall also that Carnot groups can always be "lifted" to free groups (see [28] and [6], Chapter 17). For our purposes, the main property of free Carnot groups relies on the fact that intrinsic 1-forms and 2-forms on free groups have all the same weight (see Theorem 7.2). This helps at several steps of the proofs.

We stress that several statements below and, in particular, the main result Theorem 5.1 still hold in larger classes of Carnot groups (see, for instance, Remark 6.1). However, for sake of simplicity, we do not make our intermediate statements as sharp as possible.

Proposition 4.4 Let $\mathbb{G}$ be a free Carnot group of step 2. If $\Pi_{E}$ denotes the lifting operator from the complex $\left(E_{0}^{*}, d_{c}\right)$ to $(E, d)$ defined in the Appendix, Theorem 7.1, the linear map

$$
\alpha \longrightarrow t\left(\Pi_{E} \alpha\right)
$$

is well defined and continuous from $W_{\mathbb{G}}^{2,2}\left(\mathcal{U}, E_{0}^{h}\right)$ to $L^{2}\left(\partial \mathcal{U}, \bigwedge^{h} \mathfrak{g}\right)$.

Proof. The assertion follows from Proposition 4.3, since $\Pi_{E}$ is an operator of order 1 in the horizontal derivatives, by Theorem 7.1 in the Appendix.

Now, we can define precisely our intrinsic functional. 
Definition 4.3 We define a functional in $W_{\mathbb{G}}^{2,2}\left(\mathcal{U}, \bigwedge^{1} \mathfrak{g}\right)$ as follows:

$$
J(\alpha)=\left\{\begin{array}{c}
\int_{\mathcal{U}}\left|d_{c} \alpha\right|^{2} d V+\int_{\mathcal{U}}\left|\delta_{c} \alpha\right|^{2} d V+C \int_{\mathcal{U}}|\alpha|^{2} d V \\
\quad \text { if } \alpha \in W_{\mathbb{G}}^{2,2}\left(\mathcal{U}, E_{0}^{1}\right) \text { and } t\left(\Pi_{E} \alpha\right)=0 \\
+\infty \quad \text { otherwise. }
\end{array}\right.
$$

Remark 4.1 The boundary condition $t\left(\Pi_{E} \alpha\right)=0$ il motivated by Green formula of Theorem 4.2 at the end of this Section.

Maxwell's equations, as well as the associated functional (3.14), depend on the scalar product on the fibers of $\bigwedge^{*} T \mathbb{G}$ (as for the equations, through the Hodge operator $*$ ). In fact, they are invariant under the action of Lorentz transformations for the Minkowskian metric associated with the scalar product. But it is important to point out that classical Maxwell's equations are associated with the Euclidean scalar product on the fibers $\langle\cdot, \cdot\rangle_{\text {Euc }}$, whereas group Maxwell's equations are associated with $\langle\cdot, \cdot\rangle$.

Thus, if we want to see intrinsic Maxwell's equations as a limit of classical Maxwell's equations in strongly anisotropic media, we have to take into account the change of the scalar product through a change of coordinates in the tangent bundle $\bigwedge_{1} T \mathbb{G}=T \mathbb{G}$.

Remark 4.2 In particular, though the exterior differential does not depend on the coordinates, the codifferential does depend on the scalar product. Thus, from now on, we denote by $\delta$ the codifferential associated with the Euclidean scalar product $\langle\cdot, \cdot\rangle_{\text {Euc }}$, and by $\delta_{\mathrm{g}}$ the codifferential associated with the intrinsic scalar product $\langle\cdot, \cdot\rangle$. We recall that $\delta_{c}$ denotes instead the intrinsic codifferential, associated obviously with the intrinsic scalar product.

Definition 4.4 We define the linear map

$$
R: \bigwedge_{1} T \mathbb{G} \longrightarrow \bigwedge_{1} T \mathbb{G} \quad\left\langle\Lambda_{1} R u, v\right\rangle_{\mathrm{Euc}}=\langle u, v\rangle,
$$

for any $u, v \in \Omega_{1}$. Obviously, if we set $R^{h}:=\Lambda^{h} R$, then

$$
\left\langle R^{h} \alpha, \beta\right\rangle_{\mathrm{Euc}}=\langle\alpha, \beta\rangle
$$

for any $\alpha, \beta \in \Omega^{h}$.

If $p \in \mathbb{G}$, denote now by $[T]_{p}=\left(T_{i j}(p)\right)_{i j}$ the matrix of the identity map in $\mathbb{R}^{n}$ with respect to the bases $\left\{e_{1}, \ldots, e_{n}\right\}$ and $\left\{X_{1}, \ldots, X_{n}\right\}$, i.e. $[T]_{p}$ satisfies

$$
X_{j}(p)=\sum_{i} T_{i j}(p) e_{i}, \quad j=1, \ldots, n
$$

Remark 4.3 $[T]_{p}$ coincides with $\left[\left(d t_{-p}\right)(p) \circ\left(d \tau_{p}\right)(e)\right]$, the matrix of $\left(d t_{-p}\right)(p) \circ\left(d \tau_{p}\right)(e)$ : $\bigwedge_{1} T \mathbb{G} \rightarrow \bigwedge_{1} T \mathbb{G}$ with respect to the basis $\left\{e_{1}, \ldots, e_{n}\right\}$.

It is well known that $\operatorname{det}[T]=1$ (see, e.g., [16], Proposition 2.2).

As customary, from now on we drop the index $p$ when there is no ambiguity. 
Proposition 4.5 If $\alpha \in \Omega^{h}$, then

$$
\alpha_{\mathfrak{E}}=\left[\Lambda^{h} T\right] \alpha_{\mathfrak{g}} .
$$

An analogous assertion follows if we replace covectors by vectors.

Moreover, the matrix of $R$ with respect to the Euclidean coordinates of $\mathrm{g}$ is given by

$$
[R]^{-1}=[T] \cdot[T]^{t} .
$$

In particular, by Remark 4.3, $\operatorname{det}[R]=1$.

Proof. Denote by $\theta_{p}^{j}$ and $\theta_{p}^{j, \text { Euc }}$ the $j$-element of the basis $\Theta_{p}^{h}$ and of $\Theta_{p}^{\text {Euc }}$, respectively. By Remark 4.3, we have

$$
\begin{aligned}
\alpha_{\mathfrak{g}, j}(p) & =\left\langle\alpha, \theta_{p}^{j}\right\rangle_{p}=\left\langle\Lambda^{h} d \tau_{p}(e) \alpha, \theta_{e}^{j}\right\rangle_{e} \\
& =\left\langle\Lambda^{h} d t_{-p}(p) \Lambda^{h} d \tau_{p}(e) \alpha, \theta_{p}^{j, \text { Euc }}\right\rangle_{\text {Euc }, p} \\
& =\left(\Lambda^{h} d t_{-p}(p) \Lambda^{h} d \tau_{p}(e) \alpha\right)_{\tilde{E}, j}(p) .
\end{aligned}
$$

Then assertion (4.20) follows.

The identification of $\mathfrak{g}$ and $T \mathbb{G}_{e}$ induces a stratification of $T \mathbb{G}_{e}=\wedge_{1} T \mathbb{G}_{e}$ given by $\bigwedge_{1} T \mathbb{G}_{e}=\bigwedge_{1} V_{1} \oplus \bigwedge_{1} V_{2}$. Hence, by left translation, we define also two fiber bundles on $\mathrm{G}, \bigwedge_{1} V_{i}, i=1,2$.

Definition 4.5 If $r>0$, we denote by $C_{r}: \bigwedge_{1} T \mathbb{G} \rightarrow \bigwedge_{1} T \mathbb{G}$ the linear bundle map defined on a generic fiber by

$$
C_{r}\left(X_{\ell}\right):=r^{j} X_{\ell} \quad \text { if } X_{\ell} \in \bigwedge_{1} V_{j}, \quad j=1,2 .
$$

Notice $\left(\Lambda^{2} C_{r}\right) \theta^{i} \wedge \theta^{j}=r^{w(i)+w(j)} \theta^{i} \wedge \theta^{j}$ if $w(i), w(j)$ are the weights of the $\theta^{i}, \theta^{j} \in \Lambda^{1} \mathfrak{g}$, respectively.

We denote by $\left[C_{r}\right]_{\mathfrak{g}}$ the (diagonal) matrix associate with $C_{r}$ with respect to the basis $\left\{X_{1}, \ldots, X_{n}\right\}$.

Lemma 4.1 If $r>0$, let $d_{r}$ be the "weighted exterior differential"

$$
d_{r}:=d_{0}+r d_{1}+r^{2} d_{2} .
$$

Then

$$
d_{r} \alpha=\left(\Lambda^{2} C_{r}\right) d\left(\Lambda^{1} C_{r}^{-1}\right) \alpha
$$

for any $\alpha \in \Omega^{1}$.

Proof. Choose $\alpha=\alpha_{i} \theta^{i}$. Then

$$
d_{r} \alpha=\sum_{j=0}^{2} r^{j} d_{j}\left(\alpha_{i} \theta^{i}\right)=\sum_{j=0}^{2} r^{j+w(i)} d_{j}\left(r^{-w(i)} \alpha_{i} \theta^{i}\right)=\left(\Lambda^{2} C_{r}\right) d\left(\Lambda^{1} C_{r}^{-1}\right) \alpha .
$$


Proposition 4.6 Let $r>0$ be given. Let us choose

$$
\begin{gathered}
\mu_{r}=r^{-2(Q-3) /(n-2)} C_{r}^{*} R C_{r}, \\
\varepsilon_{r}=r^{-1}\left(R C_{r}\right)^{*}
\end{gathered}
$$

where $C_{r}^{*}$ and $\left(R C_{r}\right)^{*}$ are the adjoint maps of $C_{r}$ and $R C_{r}$, respectively, with respect to the Euclidean scalar product. Then, if $\alpha \in W_{\mathbb{G}}^{2,2}\left(\mathcal{U}, \wedge^{1} \mathfrak{g}\right)\left(\subset W^{1,2}\left(\mathcal{U}, \bigwedge^{1} \mathfrak{g}\right)\right)$,

$$
\begin{aligned}
& r^{-4}\left|d_{r} \alpha\right|^{2}=\left\langle M_{r} d N_{r} R \alpha, d N_{r} R \alpha\right\rangle_{\mathrm{Euc}}, \\
& r\left\langle C_{r}^{-1} \alpha, \alpha\right\rangle=\left\langle N_{r} \Lambda^{1} R \alpha, \Lambda^{1} R \alpha\right\rangle_{\mathrm{Euc}},
\end{aligned}
$$

where $M_{r}:=\left(\operatorname{det}\left[\mu_{r}\right]\right)^{-1} \cdot \Lambda^{2} \mu_{r}$, and $N_{r}:=\Lambda^{1}\left(\varepsilon_{r}^{*}\right)^{-1}$.

Proof. Keeping in mind 4.1, we have:

$$
\begin{aligned}
\left|d_{r} \alpha\right|^{2} & =\left\langle\Lambda^{2}\left(R C_{r}\right) d\left(\Lambda^{1} C_{r}^{-1}\right) \alpha,\left(\Lambda^{2} C_{r}\right) d\left(\Lambda^{1} C_{r}^{-1}\right) \alpha\right\rangle_{\text {Euc }} \\
& =\left\langle\left(\Lambda^{2}\left(R C_{r}\right)\right) d \Lambda^{1}\left(R C_{r}\right)^{-1} R \alpha,\left(\Lambda^{2}\left(C_{r}\right)\right) d \Lambda^{1}\left(R C_{r}\right)^{-1} R \alpha\right\rangle_{\text {Euc }} \\
& =\left\langle\Lambda^{2}\left(C_{r}^{*} R C_{r}\right) d \Lambda^{1}\left(R C_{r}\right)^{-1} R \alpha, d \Lambda^{1}\left(R C_{r}\right)^{-1} R \alpha\right\rangle_{\text {Euc }} .
\end{aligned}
$$

Then assertion (4.21) follows since $\left(\Lambda^{2} \sigma L\right)=\sigma^{2}\left(\Lambda^{2} L\right)$ for any linear map $L$ and for any $\sigma \in \mathbb{R}$, and

$$
\operatorname{det} \mu_{r}=r^{2(3 n-2 Q) /(n-2)} .
$$

This proves (4.21). Identity (4.22) follows analogously.

If in the "real world" functional $\tilde{J}^{\mu, \varepsilon}$ in (3.14) we choose $\mu=\mu_{r}, \varepsilon=\varepsilon_{r}$ as above, we obtain a sequence of functionals $\left(\tilde{J}^{\mu_{r}, \varepsilon_{r}}\right)_{r>0}$ with boundary conditions $t\left(N_{r} \alpha\right)=0$.

As already pointed out, the functionals $\tilde{J}^{\mu_{r}, \varepsilon_{r}}$ contain all the information about Maxwell's equations in the matter. Now it is clear in what sense we can think of intrinsic Maxwell's equations in Carnot groups as limits of usual Maxwell's equations for anisotropic media: the Euclidean "energy" functionals $\tilde{J}^{\mu_{r}, \varepsilon_{r}} \Gamma$-converge to "energy" functional associated with the intrinsic Maxwell's equations in the group as in Definition 4.3.

However, at this point it is important to notice that this convergence has a meaning provided the functionals are all written in the same coordinates, i.e. through the same trivialization. Because of the privileged role in our approach of the limit functional, we choose to write all functionals in an intrinsic way. Therefore, through the map $R^{1}$, we write $\tilde{J}^{\mu_{r}, \varepsilon_{r}}$ in the trivialization associated with group translations, even though its "physical" meaning appears when we write $\tilde{J}^{\mu_{r}, \varepsilon_{r}}$ through the usual Euclidean trivialization.

Definition 4.6 With the notations of Proposition 4.6, if $r>0$, we denote by $J^{r}$ the functional in $W_{\mathbb{G}}^{2,2}\left(\mathcal{U}, \wedge^{1} \mathfrak{g}\right)$

$$
J^{r}(\alpha):=\left\{\begin{array}{lr}
J^{\mu_{r}, \varepsilon_{r}}\left(R^{1} \alpha\right) & \text { if } t\left(N_{r} R^{1} \alpha\right)=0 \text { on } \partial \mathcal{U} \\
+\infty & \text { otherwise }
\end{array}\right.
$$


In other words, $J^{r}$ is the variational functional associated with the classical Maxwell's equations in $\mathbb{R}^{n}$ for an anisotropic medium with magnetic permeability $\left[\mu_{r}\right]$ and dielectric permittivity $\left[\varepsilon_{r}\right]$ given by

$$
\begin{gathered}
{\left[\mu_{r}\right]=r^{-2(Q-3) /(n-2)}\left[T^{-1}\right]^{t} \cdot\left[C_{r}\right]_{\mathfrak{g}}^{2} \cdot\left[T^{-1}\right],} \\
{\left[\varepsilon_{r}\right]=r^{-1}\left[T^{-1}\right]^{t} \cdot\left[C_{r}\right]_{\mathfrak{g}} \cdot\left[T^{-1}\right] .}
\end{gathered}
$$

Proposition 4.7 If $\alpha \in W_{\mathbb{G}}^{2,2}\left(\mathcal{U}, \bigwedge^{1} \mathfrak{g}\right)$, we have

i) $\delta_{\mathrm{g}}(\alpha)=\delta\left(R^{1} \alpha\right)$;

ii) $J^{r}(\alpha)=r^{-4} \int_{\mathcal{U}}\left|d_{r} \alpha\right|^{2} d V+\int_{\mathcal{U}}\left|\delta_{\mathfrak{g}} \alpha\right|^{2} d V+C r \int_{\mathcal{U}}\left\langle C_{r}^{-1} \alpha, \alpha\right\rangle d V$ when $J^{r}(\alpha)<\infty$.

Proof. Assertion i) is a trivial consequence of the definition of $R$. Assertion ii) follows by Proposition 4.6.

We conclude this Section going back to the condition $t\left(\Pi_{E} \alpha\right)=0$ that is justified by the following Green formula. We stress that this formula is not a straightforward consequence of Stokes theorem as in $\mathbb{R}^{n}$. Indeed, the identity $d_{c}(\alpha \wedge \beta)=d_{c} \alpha \wedge \beta-\alpha \wedge d_{c} \beta$ fails to hold for intrinsic forms, as pointed out in [4], Proposition A.7, since $\alpha \wedge \beta$ cannot be defined in a coherent way.

Theorem 4.2 (Green formula) Let $\mathbb{G}$ be a Carnot group of arbitrary step. If $\alpha \in C^{\infty}\left(\overline{\mathcal{U}}, E_{0}^{h+1}\right)$, $0 \leq h \leq n-1$, and $\beta \in C^{\infty}\left(\overline{\mathcal{U}}, E_{0}^{h}\right)$, we have

$$
\int_{\mathcal{U}}\left\langle\alpha, d_{c} \beta\right\rangle d V=\int_{\mathcal{U}}\left\langle\delta_{c} \alpha, \beta\right\rangle d V+\int_{\partial \mathcal{U}} t\left(\Pi_{E} \beta\right) \wedge * n(\alpha)
$$

Proof. First of all, we notice the following identity holds:

$$
\Pi_{E_{0}} \Pi_{E}+\Pi_{F}\left(I-\Pi_{E_{0}} \Pi_{E}\right)=I .
$$

Indeed, by Theorem 7.1, $I=\Pi_{E}+\Pi_{F}=\Pi_{E} \Pi_{E_{0}} \Pi_{E}+\Pi_{F}=\Pi_{E_{0}} \Pi_{E}-\Pi_{F} \Pi_{E_{0}} \Pi_{E}+\Pi_{F}=$ $\Pi_{E_{0}} \Pi_{E}+\Pi_{F}\left(I-\Pi_{E_{0}} \Pi_{E}\right)$. 
Then, by classical Green formula (see e.g. [21], Chapter 5, Section 2.6, Proposition 2),

$$
\begin{aligned}
& \int_{\mathcal{U}}\left\langle\alpha, d_{c} \beta\right\rangle d V-\int_{\partial \mathcal{U}} t\left(\Pi_{E} \beta\right) \wedge * n(\alpha) \\
& =\int_{\mathcal{U}}\left\langle\alpha, \Pi_{E_{0}} d \Pi_{E} \beta\right\rangle d V-\int_{\partial \mathcal{U}} t\left(\Pi_{E} \beta\right) \wedge * n(\alpha) \\
& =\int_{\mathcal{U}}\left\langle\alpha, d \Pi_{E} \beta\right\rangle d V-\int_{\partial \mathcal{U}} t\left(\Pi_{E} \beta\right) \wedge * n(\alpha) \quad\left(\text { since } \alpha \in E_{0}^{h+1}\right) \\
& =\int_{\mathcal{U}}\left\langle\delta \alpha, \Pi_{E} \beta\right\rangle d V \\
& =\int_{\mathcal{U}}\left\langle\delta \alpha, \Pi_{E} \beta\right\rangle d V=(-1)^{h(n-h)} \int_{\mathcal{U}}\left\langle * * \delta \alpha, \Pi_{E} \beta\right\rangle d V \\
& =(-1)^{h(n-h)} \int_{\mathcal{U}}\left\langle * \Pi_{E_{0}} \Pi_{E} * \delta \alpha, \Pi_{E} \beta\right\rangle d V \\
& +(-1)^{h(n-h)} \int_{\mathcal{U}}\left\langle * \Pi_{F}\left(I-\Pi_{E_{0}} \Pi_{E}\right) * \delta \alpha, \Pi_{E} \beta\right\rangle d V \quad \text { (by (4.26)) } \\
& =(-1)^{h(n-h)} \int_{\mathcal{U}}\left\langle * \Pi_{E_{0}} \Pi_{E} * \delta \alpha, \Pi_{E} \beta\right\rangle d V \quad \text { (by Lemma 7.4) } \\
& =(-1)^{h(n-h+1)+1} \int_{\mathcal{U}}\left\langle * \Pi_{E_{0}} \Pi_{E} d * \alpha, \Pi_{E} \beta\right\rangle d V \\
& =(-1)^{h(n-h+1)+1} \int_{\mathcal{U}}\left\langle * \Pi_{E_{0}} d \Pi_{E} * \alpha, \Pi_{E} \beta\right\rangle d V \\
& =(-1)^{h(n-h+1)+1} \int_{\mathcal{U}}\left\langle * d_{c} * \alpha, \Pi_{E} \beta\right\rangle d V \\
& =\int_{\mathcal{U}}\left\langle\delta_{c} \alpha, \Pi_{E} \beta\right\rangle d V=\int_{\mathcal{U}}\left\langle\Pi_{E_{0}} \delta_{c} \alpha, \Pi_{E} \beta\right\rangle d V \quad\left(\text { since } \delta_{c} \alpha \in E_{0}^{h}\right) \\
& =\int_{\mathcal{U}}\left\langle\delta_{c} \alpha, \Pi_{E_{0}} \Pi_{E} \Pi_{E_{0}} \beta\right\rangle d V \quad \text { (since } \beta \in E_{0}^{h} \text { ) } \\
& =\int_{\mathcal{U}}\left\langle\delta_{c} \alpha, \Pi_{E_{0}} \beta\right\rangle d V \\
& =\int_{\mathcal{U}}\left\langle\delta_{c} \alpha, \beta\right\rangle d V \quad \text { (again since } \beta \in E_{0}^{h} \text { ). }
\end{aligned}
$$

Thus (4.25) is proved.

\section{Main results}

In this section we state our main convergence result. We briefly recall the definition of $\Gamma$-convergence. 
Definition 5.1 (see, e.g. [10], Chapter 4) Let $X$ be a topological space, and denote by $\mathcal{N}(x)$ the family of all open neighborhoods of $x$ in $X$. If $h \in \mathbb{N}$, let

$$
J_{h}: X \longrightarrow[-\infty,+\infty]
$$

be functionals on $X$. We set

$$
\left(\Gamma-\liminf _{h \rightarrow \infty} J_{h}\right)(x)=\sup _{V \in \mathcal{N}(x)} \liminf _{h \rightarrow \infty} \inf _{y \in V} J_{h}(y),
$$

and

$$
\left(\Gamma-\limsup _{h \rightarrow \infty} J_{h}\right)(x)=\sup _{V \in \mathcal{N}(x)} \limsup _{h \rightarrow \infty} \inf _{y \in V} J_{h}(y) .
$$

If there exists $J: X \longrightarrow[-\infty,+\infty]$ such that

$$
\Gamma-\liminf _{h \rightarrow \infty} J_{h}=\Gamma-\limsup _{h \rightarrow \infty} J_{h}=J,
$$

then we say that $\left\{J_{h}\right\}_{h \in \mathbb{N}} \Gamma$-converges to $J$ in $X$, and we write

$$
J=\Gamma-\lim _{h \rightarrow \infty} J_{h}
$$

In metric spaces we have the following characterization of $\Gamma$-convergence.

Proposition 5.1 (see, e.g., [10], Proposition 8.1) Let $X$ be a metric space, and let

$$
J_{r}, J: X \longrightarrow[-\infty,+\infty]
$$

with $r>0$ be functionals on $X$. Then $\left\{J_{r}\right\}_{r>0} \Gamma$-converges to $J$ on $X$ as $r$ goes to zero if and only if the following two conditions hold:

1) for every $u \in X$ and for every sequence $\left\{u_{r_{k}}\right\}_{k \in \mathbb{N}}$ with $r_{k} \rightarrow 0$ as $k \rightarrow \infty$, which converges to $u$ in $X$, there holds

$$
\liminf _{k \rightarrow \infty} J_{r_{k}}\left(u_{r_{k}}\right) \geq J(u)
$$

2) for every $u \in X$ and for every sequence $\left\{r_{k}\right\}_{k \in \mathbb{N}}$ with $r_{k} \rightarrow 0$ as $k \rightarrow \infty$ there exists a subsequence (still denoted by $\left\{r_{k}\right\}_{k \in \mathbb{N}}$ ) such that $\left\{u_{r_{k}}\right\}_{k \in \mathbb{N}}$ converges to $u$ in $X$ and

$$
\limsup _{k \rightarrow \infty} J_{r_{k}}\left(u_{r_{k}}\right) \leq J(u)
$$

In a metric space, to avoid cumbersome notations, from now on we write systematically $\lim _{r \rightarrow 0}$ to mean a limit with $r=r_{k}$, where $\left\{r_{k}\right\}_{k \in \mathbb{N}}$ is any sequence with $r_{k} \rightarrow 0$ as $k \rightarrow \infty$.

For a deep and detailed survey on $\Gamma$-convergence, we refer to the monograph [10].

Proposition 5.2 Suppose $\alpha^{r} \rightarrow \alpha$ as $r \rightarrow 0$ in $W_{\mathbb{G}}^{2,2}\left(\mathcal{U}, \bigwedge^{1} \mathfrak{g}\right)$. Then

$$
J(\alpha) \leq \liminf _{r \rightarrow 0} J_{r}\left(\alpha^{r}\right) .
$$


Proof. As usual, without loss of generality, we may assume $\lim \inf _{r \rightarrow 0} J_{r}\left(\alpha^{r}\right)<\infty$.

We can argue now following the guidelines of Theorem 5.1 in [2]. First of all, we show that

$$
\int_{\mathcal{U}}\left|d_{c} \alpha\right|^{2} d V \leq \liminf _{r \rightarrow 0} r^{-4} \int_{\mathcal{U}}\left|d_{r} \alpha^{r}\right|^{2} d V .
$$

To this end, keeping in mind (7.43), we write

$$
\alpha^{r}=\alpha_{1}^{r}+\alpha_{2}^{r},
$$

with $\alpha_{i}^{r} \in \Omega^{1, i}, i=1,2$. Reordering the terms of $d_{r} \alpha^{r}$ according to their weights, we have the following orthogonal decomposition:

$$
d_{r} \alpha^{r}=\left(d_{0} \alpha_{2}^{r}+r d_{1} \alpha_{1}^{r}\right)+\left(r d_{1} \alpha_{2}^{r}+r^{2} d_{2} \alpha_{1}^{r}\right)+r^{2} d_{2} \alpha_{2}^{r} .
$$

Therefore we can write

$$
\begin{aligned}
r^{-4} \int_{\mathcal{U}}\left|d_{r} \alpha^{r}\right|^{2} d V & =r^{-4} \int_{\mathcal{U}}\left|d_{0} \alpha_{2}^{r}+r d_{1} \alpha_{1}^{r}\right|^{2} d V \\
& +r^{-2} \int_{\mathcal{U}}\left|d_{1} \alpha_{2}^{r}+r d_{2} \alpha_{1}^{r}\right|^{2} d V \\
& +\int_{\mathcal{U}}\left|d_{2} \alpha_{2}^{r}\right|^{2} d V
\end{aligned}
$$

Since $\liminf \operatorname{in}_{r \rightarrow 0} J_{r}\left(\alpha^{r}\right)<\infty$, we have

- if $r \in(0,1)$,

$$
\left.r^{-1}\left(d_{1} \alpha_{2}^{r}+r d_{2} \alpha_{1}^{r}\right) \quad \text { is uniformly bounded in } L^{2}\left(\mathcal{U}, \bigwedge^{2} \mathfrak{g}\right)\right)
$$

- if $r \rightarrow 0$, then

$$
\left.d_{0} \alpha_{2}^{r}+r d_{1} \alpha_{1}^{r}=O\left(r^{2}\right) \text { in } L^{2}\left(\mathcal{U}, \bigwedge^{2} \mathfrak{g}\right)\right)
$$

Now, (5.30) yields eventually that

$$
d_{0} \alpha_{2}=0
$$

since $d_{0}$ is algebraic and $\left\{\alpha^{r}\right\}_{0<r<1}$ is bounded in $W_{\mathbb{G}}^{2,2}\left(\mathcal{U}, \wedge^{1} \mathfrak{g}\right)$. Thus, keeping in mind that $d_{0}\left(\bigwedge^{1,1} \mathfrak{g}\right)=\{0\}$, we can conclude that $\alpha \in \operatorname{ker} d_{0}=E_{0}^{1}$, and therefore $\alpha=\alpha_{1}$.

Because of convergence of $\left(\alpha^{r}\right)_{r>0}$ in $W_{\mathbb{G}}^{2,2}\left(\mathcal{U}, \wedge^{1} \mathfrak{g}\right)$ when $r \rightarrow 0$, it follows that

$$
\alpha_{1}^{r} \rightarrow \alpha_{1}:=\left(\Pi_{E} \alpha\right)_{1}
$$

in $L^{2}\left(\mathcal{U}, \bigwedge^{1} \mathfrak{g}\right)$ as $r \rightarrow 0$ as well as

$$
d_{0}^{-1} d_{1} \alpha_{1}^{r} \rightarrow d_{0}^{-1} d_{1} \alpha_{1}:=-\left(\Pi_{E} \alpha\right)_{2} \quad \text { in } L^{2}\left(\mathcal{U}, \bigwedge^{1} \mathfrak{g}\right),
$$


since $d_{1}$ is an homogeneous differential operator in the horizontal derivatives of order 1 and $d_{0}^{-1}$ is algebraic. By (5.30), we have now

$$
d_{0} \alpha_{2}^{r}+r d_{1} \alpha_{1}^{r}=O\left(r^{2}\right) \text { in } L^{2}\left(\mathcal{U}, \bigwedge^{2} \mathfrak{g}\right) .
$$

On the other hand, by Lemma 7.3, ii), $d_{0}^{-1} d_{0} \alpha_{2}^{r}=\alpha_{2}^{r}$, since $\alpha_{2}^{r}$ has weight 2 and hence is orthogonal to $\Omega^{1,1}=\operatorname{ker} d_{0}$. Thus, keeping in mind that $d_{0}^{-1}$ is algebraic, it follows from (5.33) that

$$
\frac{1}{r} \alpha_{2}^{r}+d_{0}^{-1} d_{1} \alpha_{1}^{r}=O(r) \quad \text { in } L^{2}\left(\mathcal{U}, \bigwedge^{1} \mathfrak{g}\right),
$$

and therefore

$$
\frac{1}{r} \alpha_{2}^{r} \rightarrow\left(\Pi_{E} \alpha\right)_{2} \quad \text { in } L^{2}\left(\mathcal{U}, \bigwedge^{1} \mathfrak{g}\right)
$$

Now, by (5.31) and (5.32), we obtain

$$
\frac{1}{r}\left(d_{1} \alpha_{2}^{r}+r d_{2} \alpha_{1}^{r}\right) \longrightarrow d_{1}\left(\Pi_{E} \alpha\right)_{2}+d_{2}\left(\Pi_{E} \alpha\right)_{1}
$$

as $r \rightarrow 0$ in the sense of distributions. On the other hand, the limit $d_{1}\left(\Pi_{E} \alpha\right)_{2}+d_{2}\left(\Pi_{E} \alpha\right)_{1}$ belongs to $L^{2}\left(\mathcal{U}, \bigwedge^{2} \mathfrak{g}\right.$ ) (since $d_{\ell}\left(\Pi_{E} \omega\right)_{3-\ell}$ is an homogeneous differential operator in the horizontal derivatives of order 2 , by Theorem 7.1, i) and Definition 7.2), and

$$
\left\{\frac{1}{r}\left(d_{1} \alpha_{2}^{r}+r d_{2} \alpha_{1}^{r}\right)\right\}_{r>0} \text { is equibounded in } L^{2}\left(\mathcal{U}, \bigwedge^{2} \mathfrak{g}\right),
$$

as $r \rightarrow 0$, by (5.29). Combining (5.35) and (5.34) we obtain that the limit in (5.34) is in fact a weak limit in $L^{2}\left(\mathcal{U}, \bigwedge^{2} \mathfrak{g}\right)$ Thus, if we keep in mind Theorem 7.2, we obtain

$$
\begin{aligned}
& \int_{\mathcal{U}}\left|d_{c} \alpha\right|^{2} d V=\int_{\mathcal{U}}\left|\Pi_{E_{0}}\left(d_{1}\left(\Pi_{E} \alpha\right)_{2}+d_{2}\left(\Pi_{E} \alpha\right)_{1}\right)\right|^{2} d V \\
& \leq \int_{\mathcal{U}}\left|\left(d_{1}\left(\Pi_{E} \alpha\right)_{2}+d_{2}\left(\Pi_{E} \alpha\right)_{1}\right)\right|^{2} d V \\
& \quad \leq \liminf _{r \rightarrow 0} r^{-2} \int_{\mathcal{U}}\left|d_{1} \alpha_{2}^{r}+r d_{2} \alpha_{1}^{r}\right|^{2} d V \leq \liminf _{r \rightarrow 0} r^{-4} \int_{\mathcal{U}}\left|d_{r} \alpha^{r}\right|^{2} d V .
\end{aligned}
$$

This proves (5.28).

Let us consider now the divergence term in $J_{r}\left(\alpha^{r}\right)$. Since $\liminf _{r \rightarrow 0} J_{r}\left(\alpha^{r}\right)<\infty$, we have that

$$
\left(\delta_{g} \alpha^{r}\right)_{r>0} \quad \text { is uniformly bounded in } L^{2}(\mathcal{U}) .
$$

On the other hand, we can write $\delta_{g} \alpha^{r}$ as

$$
\delta_{g} \alpha^{r}=\delta_{g} \alpha_{1}^{r}+\delta_{g} \alpha_{2}^{r},
$$

where, for $i=1,2, \delta_{g} \alpha_{i}^{r}$ is a sum of terms of the form $X_{\ell}\left(\alpha^{r}\right)_{\ell}$, where $\left(\alpha^{r}\right)_{\ell}$ is the $\ell$-th component of $\alpha^{r}$ with respect to the basis $\theta_{1}, \ldots, \theta_{n}$, and $X_{\ell} \in V_{i}$. But $\delta_{g} \alpha_{1}^{r} \rightarrow \delta_{g} \alpha_{1}=\delta_{c} \alpha$ in $L^{2}(\mathcal{U})$ (since $\delta_{g} \alpha_{1}^{r}$ contains only first order horizontal derivatives), and therefore $\delta_{g} \alpha_{2}^{r}$ 
weakly converge in $L^{2}(\mathcal{U})$. On the other hand, $\delta_{g} \alpha_{2}^{r} \rightarrow \delta_{g} \alpha_{2}=0$ in the sense of distributions, since $\alpha^{r} \rightarrow \alpha$ in $L^{2}(\mathcal{U})$, and $\alpha$ is horizontal (as seen in (5.31)). Thus, eventually,

$$
\delta_{g} \alpha^{r} \rightarrow \delta_{c} \alpha \quad \text { weakly in } L^{2}(\mathcal{U}),
$$

and therefore

$$
\int_{\mathcal{U}}\left|\delta_{c} \alpha\right|^{2} d V \leq \liminf _{r \rightarrow 0} \int_{\mathcal{U}}\left|\delta_{g} \alpha^{r}\right|^{2} d V .
$$

On the other hand, if we split again $\alpha^{r}$ gathering the terms by their weights (i.e. $\alpha^{r}=$ $\alpha_{1}^{r}+\alpha_{2}^{r}$, with $\left.\alpha_{j}^{r} \in \Omega^{1, j}\right)$, keeping in mind that $\alpha_{1}^{r} \rightarrow \alpha_{1}$ in $L^{2}$, we have

$$
\begin{aligned}
\liminf _{r \rightarrow 0} & \int_{\mathcal{U}} r\left\langle C_{r}^{-1} \alpha^{r}, \alpha^{r}\right\rangle d V=\liminf _{r \rightarrow 0}\left(\int_{\mathcal{U}}\left|\alpha_{1}^{r}\right|^{2} d V+\frac{1}{r} \int_{\mathcal{U}}\left|\alpha_{2}^{r}\right|^{2} d V\right) \\
\geq & \liminf _{r \rightarrow 0} \int_{\mathcal{U}}\left|\alpha_{1}^{r}\right|^{2} d V=\int_{\mathcal{U}}\left|\alpha_{1}\right|^{2} d V=\int_{\mathcal{U}}|\alpha|^{2} d V,
\end{aligned}
$$

since $\alpha \in E_{0}^{1}$.

Summing up (5.28), (5.37), and (5.38), in order to obtain equation (5.27), we have but to prove that $t\left(\Pi_{E} \alpha\right)=0$. This follows straightforwardly since $0=t\left(N_{r} R^{1} \alpha^{r}\right)=r t\left(C_{1 / r} \alpha^{r}\right)=$ $t\left(\alpha_{1}^{r}+r^{-1} \alpha_{2}^{r}\right)$, and then $t\left(\alpha_{1}^{r}+r^{-1} \alpha_{2}^{r}\right)=0$. But, by Proposition 4.4, $t\left(\alpha_{1}^{r}+r^{-1} \alpha_{2}^{r}\right)$ weakly converge to $t\left(\Pi_{E} \alpha\right)$ in the space of 1 -forms on $\partial \mathcal{U}$, endowed with the $L^{2}$-norm with respect to the measure $d \sigma$, as defined in (4.19). This achieves the proof of (5.27).

Definition 5.2 If $\alpha \in \Omega^{1}(\mathcal{U})$, keeping in mind (7.43), we write

$$
\alpha=\alpha_{1}+\alpha_{2}
$$

with $\alpha_{i} \in \Omega^{1, i}(\mathcal{U}), i=1,2$. If $m \geq 2$, we say that

$$
\alpha \in \widehat{W}_{\mathbb{G}}^{m, 2}\left(\mathcal{U}, \bigwedge^{1} \mathfrak{g}\right) \quad \text { iff } \quad \alpha_{i} \in W_{\mathbb{G}}^{m+1-i, 2}\left(\mathcal{U}, \bigwedge^{1} \mathfrak{g}\right), \quad i=1,2
$$

The space $\widehat{W}_{\mathbb{G}}^{m, 2}\left(\mathcal{U}, \bigwedge^{1} \mathfrak{g}\right)$ is endowed with its natural norm.

Remark 5.1 Any horizontal form in $\widehat{W}_{\mathbb{G}}^{2,2}\left(\mathcal{U}, \wedge^{1} \mathfrak{g}\right)$ belongs to $W_{\mathbb{G}}^{2,2}\left(\mathcal{U}, E_{0}^{1}\right)$. In addition, the map $\alpha \rightarrow \Pi_{E} \alpha$ is continuous from $W_{\mathbb{G}}^{2,2}\left(\mathcal{U}, E_{0}^{1}\right)$ to $\widehat{W}_{\mathbb{G}}^{2,2}\left(\mathcal{U}, \wedge^{1} \mathfrak{g}\right)$.

Proposition 5.3 Notice that $\widehat{W}^{3,2}\left(\mathcal{U}, \wedge^{1} \mathfrak{g}\right) \cap L^{2}\left(\mathcal{U}, E_{0}^{1}\right)=W_{\mathbb{G}}^{3,2}\left(\mathcal{U}, E_{0}^{1}\right)$, and let $\alpha \in W_{\mathbb{G}}^{3,2}\left(\mathcal{U}, E_{0}^{1}\right)$ be such that $t\left(\Pi_{E} \alpha\right)=0$. Then there exists a sequence $\left(\alpha^{r}\right)_{r>0}$ in $\widehat{W}_{\mathbb{G}}^{3,2}\left(\mathcal{U}, \wedge^{1} \mathfrak{g}\right)$ such that
i) $\alpha^{r} \rightarrow \alpha$ in $W_{\mathbb{G}}^{2,2}\left(\mathcal{U}, \bigwedge^{1} \mathfrak{g}\right)$;
ii) $J_{r}\left(\alpha^{r}\right) \rightarrow J(\alpha)$ as $r \rightarrow 0$.

Proof. Arguing as in [2] (dropping however the reduction argument), we choose

$$
\alpha^{r}=\alpha+r\left(\Pi_{E} \alpha\right)_{2} .
$$


First of all, $\left(\Pi_{E} \alpha\right)_{2} \in W_{\mathbb{G}}^{2,2}\left(\mathcal{U}, \wedge^{1} \mathfrak{g}\right)$, since $\Pi_{E}$ is an operator of order 1 in the horizontal derivatives, and hence i) follows trivially. Moreover

$$
t\left(N_{r} R^{1} \alpha^{r}\right)=\operatorname{rt}\left(C_{1 / r} \alpha^{r}\right)=t\left(\alpha+\left(\Pi_{E} \alpha\right)_{2}\right)=t\left(\Pi_{E} \alpha\right)=0 .
$$

Thus, in order to prove ii), we have but to show that

$$
J_{r}\left(\alpha^{r}\right) \rightarrow J(\alpha) \quad \text { as } r \rightarrow 0 .
$$

Arguing as in [2], we can write

$$
\begin{aligned}
d_{r} \alpha^{r} & =r\left(d_{0}\left(\Pi_{E} \alpha\right)_{2}+d_{1}\left(\Pi_{E} \alpha\right)_{1}\right)+r^{2}\left(d_{1}\left(\Pi_{E} \alpha\right)_{2}+d_{2}\left(\Pi_{E} \alpha\right)_{1}\right) \\
& +r^{3} d_{2}\left(\Pi_{E} \alpha\right)_{2} .
\end{aligned}
$$

Notice also that

$$
d_{0}\left(\Pi_{E} \alpha\right)_{2}+d_{1}\left(\Pi_{E} \alpha\right)_{1}=-d_{0} d_{0}^{-1} d_{1} \alpha+d_{1} \alpha=0,
$$

since $d_{0}^{-1} d_{0}=I d$ on $\mathcal{R}\left(d_{0}\right)$, and $d_{1} \alpha \in \mathcal{R}\left(d_{0}\right)$, by Lemma 7.5 - (1) in the Appendix.

Let us suppose for a while we know that

$$
d_{1}\left(\Pi_{E} \alpha\right)_{2}+d_{2}\left(\Pi_{E} \alpha\right)_{1} \in E_{0}^{2},
$$

in particular $\Pi_{E_{0}^{\perp}}\left(d_{1}\left(\Pi_{E} \alpha\right)_{2}+d_{2}\left(\Pi_{E} \alpha\right)_{1}\right)=0$. Thus, we can write

$$
\begin{aligned}
r^{-4} & \int_{\mathcal{U}}\left|d_{r} \alpha^{r}\right|^{2} d V \\
& =\int_{\mathcal{U}}\left|d_{1}\left(\Pi_{E} \alpha\right)_{2}+d_{2}\left(\Pi_{E} \alpha\right)_{1}\right|^{2} d V+r^{2} \int_{\mathcal{U}}\left|d_{2}\left(\Pi_{E} \alpha\right)_{2}\right|^{2} d V \\
& =\int_{\mathcal{U}} \mid\left(\left.\Pi_{E_{0}}\left(d_{1}\left(\Pi_{E} \alpha\right)_{2}+d_{2}\left(\Pi_{E} \alpha\right)_{1}\right)\right|^{2} d V+r^{2} \int_{\mathcal{U}}\left|d_{2}\left(\Pi_{E} \alpha\right)_{2}\right|^{2} d V\right. \\
& =\int_{\mathcal{U}}\left|d_{c} \alpha\right|^{2} d V+r^{2} \int_{\mathcal{U}}\left|d_{2}\left(\Pi_{E} \alpha\right)_{2}\right|^{2} d V \rightarrow \int_{\mathcal{U}}\left|d_{c} \alpha\right|^{2} d V,
\end{aligned}
$$

as $r \rightarrow 0$, since $d_{2}\left(\Pi_{E} \alpha\right)_{2} \in L^{2}\left(\mathcal{U}, \bigwedge^{2} \mathfrak{g}\right)$.

On the other hand, it is obvious that $\delta_{g} \alpha^{r} \rightarrow \delta_{c} \alpha$ in $L^{2}(\mathcal{U})$, again since the coefficients of $\left(\Pi_{E} \alpha\right)_{2}$ belong to $W_{\mathbb{G}}^{2,2}(\mathcal{U})$.

Finally

$$
\int_{\Omega} r\left\langle C_{r}^{-1} \alpha^{r}, \alpha^{r}\right\rangle d V=\int_{\Omega}|\alpha|^{2} d V+r \int_{\Omega}\left|\left(\Pi_{E} \alpha\right)_{2}\right|^{2} d V \rightarrow \int_{\Omega}|\alpha|^{2} d V
$$

as $r \rightarrow 0$. This achieves the proof of the Proposition, once (5.39) is proved. Indeed, (5.39) follows straitforwardly by the identity $d^{2}=0$. In fact, gathering all terms of the same weight, we get

$$
d_{0}^{2}=0, \quad d_{0} d_{1}=-d_{1} d_{0}, \quad d_{0} d_{2}=-d_{2} d_{0}-d_{1}^{2} .
$$


Hence

$$
d_{0}\left(d_{1}\left(\Pi_{E} \alpha\right)_{2}+d_{2}\left(\Pi_{E} \alpha\right)_{1}\right)=d_{1} d_{0} d_{0}^{-1} d_{1} \alpha-d_{1}^{2} \alpha=0,
$$

since, again by Lemma 7.5 in the Appendix, $d_{1} \alpha \in \mathcal{R}\left(d_{0}\right)$ and $d_{0}^{-1} d_{0}=I d$ on $\mathcal{R}\left(d_{0}\right)$.

Denote by $j_{r}$ and $j$ the restrictions of $J_{r}$ and $J$ to $\widehat{W}_{\mathbb{G}}^{3,2}\left(\mathcal{U}, \bigwedge^{1} \mathfrak{g}\right)$, respectively. We see below that $\left(j_{r}\right)_{r>0} \Gamma$-converges (with respect to the topology induced by $W_{\mathbb{G}}^{2,2}\left(\mathcal{U}, \wedge^{1} \mathfrak{g}\right)$ ) to $j$. However, in general, the $\Gamma$-limit of the restriction of a sequence of functionals is only greater or equal than the restrictions of both $\Gamma$ - lim sup and $\Gamma$-lim inf of the same sequence (for further details, see Proposition 6.14 in [10] and the remarks therein).

On the contrary, in our case Propositions 5.2 and 5.3 yield a more precise result.

Theorem 5.1 Let $\mathbb{G}$ be a free step 2 Carnot group. If $r>0$, denote respectively by $j_{r}$ and $j$ the restrictions of $J_{r}$ and $J$ to $\widehat{W}_{\mathbb{G}}^{3,2}\left(\mathcal{U}, \wedge^{1} \mathfrak{g}\right)$. Then

i) $\left(j_{r}\right)_{r>0} \Gamma$-converges to $j$ in $\widehat{W}_{\mathbb{G}}^{3,2}\left(\mathcal{U}, \bigwedge^{1} \mathfrak{g}\right)$ with respect to the topology induced by $W_{\mathbb{G}}^{2,2}\left(\mathcal{U}, \wedge^{1} \mathfrak{g}\right)$

ii) in $\widehat{W}_{\mathbb{G}}^{3,2}\left(\mathcal{U}, \bigwedge^{1} \mathfrak{g}\right)$ we have

$$
\Gamma-\liminf _{r \rightarrow 0} J^{r} \equiv \Gamma-\limsup _{r \rightarrow 0} J^{r} \equiv j \equiv J_{\widehat{W}_{\Theta}^{3,2}\left(\mathcal{U}, E_{0}^{1}\right)}
$$

where the $\Gamma$-limits must be meant with respect to the topology induced by $W_{\mathbb{G}}^{2,2}\left(\mathcal{U}, \wedge^{1} \mathfrak{g}\right)$.

Proof. Assertion i) follows from Propositions 5.2 and 5.3.

As for ii), let now $\alpha \in \widehat{W}_{\mathbb{G}}^{3,2}\left(\mathcal{U}, \wedge^{1} \mathfrak{g}\right)$ be given. By definition ([10], Proposition 8.1), there exists a sequence $\left(\alpha^{r}\right)_{r>0}$ in $W_{\mathbb{G}}^{2,2}\left(\mathcal{U}, \wedge^{1} \mathfrak{g}\right)$, converging to $\alpha$ in the topology of $W_{\mathbb{G}}^{2,2}\left(\mathcal{U}, \bigwedge^{1} \mathfrak{g}\right)$, such that $J^{r}\left(\alpha^{r}\right) \rightarrow\left(\Gamma-\liminf _{r \rightarrow 0} J^{r}\right)(\alpha)$ as $r \rightarrow 0$. Suppose now $\left(\Gamma-\liminf _{r \rightarrow 0} J^{r}\right)(\alpha)<\infty$. Then, by Proposition 5.2,

$$
J(\alpha) \leq \liminf _{r \rightarrow 0} J^{r}\left(\alpha^{r}\right)=\left(\Gamma-\liminf _{r \rightarrow 0} J^{r}\right)(\alpha) .
$$

Clearly, inequality (5.40) still holds trivially if $\left(\Gamma-\liminf _{r \rightarrow 0} J^{r}\right)(\alpha)=\infty$.

On the other hand, by [10], Proposition 6.14,

$$
J(\alpha) \leq \limsup _{r \rightarrow 0} J^{r}\left(\alpha^{r}\right) \leq(\alpha) \leq \limsup _{r \rightarrow 0} J^{r}\left(\alpha^{r}\right)=j(\alpha)=J(\alpha) .
$$

Combining (5.40) and (5.41) we achieve the proof of the theorem.

\section{An example: Maxwell's equation in $\mathbb{H}^{1}$}

Consider now in particular the first Heisenberg group $\mathbb{G}=\mathbb{H}^{1}$, with variables $x, y, t$. Set $X:=\partial_{x}-\frac{1}{2} y \partial_{t}, Y:=\partial_{y}+\frac{1}{2} x \partial_{t}, T:=\partial_{t}$. The stratification of the algebra $\mathfrak{g}$ is given by 
$\mathfrak{g}=V_{1} \oplus V_{2}$, where $V_{1}=\operatorname{span}\{X, Y\}$ and $V_{2}=\operatorname{span}\{T\}$. We have $X^{\natural}=d x, Y^{\natural}=d y$, $T^{\natural}=\theta:=d t+\frac{1}{2}(y d x-x d y)$ (the contact form of $\left.\mathbb{H}^{1}\right)$. In this case

$$
\begin{aligned}
& E_{0}^{1}=\operatorname{span}\{d x, d y\} \\
& E_{0}^{2}=\operatorname{span}\{d x \wedge \theta, d y \wedge \theta\} \\
& E_{0}^{3}=\operatorname{span}\{d x \wedge d y \wedge \theta\} .
\end{aligned}
$$

The action of $d_{c}$ on $E_{0}^{1}$ is given by ([29], [17], [4])

$$
\begin{aligned}
& d_{c}\left(\alpha_{1} d x+\alpha_{2} d y\right) \\
& \quad=\left(X^{2} \alpha_{2}-2 X Y \alpha_{1}+Y X \alpha_{1}\right) d x \wedge \theta+\left(2 Y X \alpha_{2}-Y^{2} \alpha_{1}-X Y \alpha_{2}\right) d y \wedge \theta \\
& \quad:=P_{1}\left(\alpha_{1}, \alpha_{2}\right) d x \wedge \theta+P_{2}\left(\alpha_{1}, \alpha_{2}\right) d y \wedge \theta .
\end{aligned}
$$

We see that $d_{c}$ is a homogeneous operator of order 2 in the horizontal derivatives.

On the other hand

$$
\delta_{c} \alpha=X \alpha_{1}+Y \alpha_{2} .
$$

Thus, if $E=E_{1} d x+E_{2} d y$ and $H=H_{1} d x+H_{2} d y$, equations (4.15) and (4.16) read as

$$
\begin{aligned}
& P_{2}\left(E_{1}, E_{2}\right) d x-P_{1}\left(E_{1}, E_{2}\right) d y=i \omega\left(H_{1} d x+H_{2} d y\right) \\
& P_{2}\left(H_{1}, H_{2}\right) d x-P_{1}\left(H_{1}, H_{2}\right) d y=-i \omega\left(E_{1} d x+E_{2} d y\right)
\end{aligned}
$$

and

$$
X H_{1}+Y H_{2}=0, \quad X E_{1}+Y E_{2}=0 .
$$

In this case

$$
[T]=\left(\begin{array}{ccc}
1 & 0 & 0 \\
0 & 1 & 0 \\
-\frac{1}{2} y & \frac{1}{2} x & 1
\end{array}\right), \quad\left[C_{r}\right]_{\mathfrak{g}}=\left(\begin{array}{ccc}
r & 0 & 0 \\
0 & r & 0 \\
0 & 0 & r^{2}
\end{array}\right) .
$$

Thus, by (4.23) and (4.24), the magnetic permeability $\left[\mu_{r}\right]$ and dielectric permittivity $\left[\varepsilon_{r}\right]$ of the "approximating equations" take the forms

$$
\left[\mu_{r}\right]=\left[\varepsilon_{r}\right]=\left(\begin{array}{ccc}
1+\frac{r}{4} y^{2} & -\frac{r}{4} x y & \frac{r}{2} y \\
-\frac{r}{4} x y & 1+\frac{r}{4} x^{2} & -\frac{r}{2} x \\
\frac{r}{2} y & -\frac{r}{2} x & r
\end{array}\right) .
$$

Remark 6.1 Heisenberg groups $\mathbb{H}^{N}$ with $N>1$ are still step 2 groups, though not free. Nevertheless, intrinsic 2-forms on $\mathbb{H}^{N}$ have weight 2, and therefore all our arguments can be carried out with the following choice of $\mu_{r}$ and $\varepsilon_{r}$ :

$$
\mu_{r}=r^{-4 N /(2 N-1)} C_{r}^{*} R C_{r},
$$

and

$$
\varepsilon_{r}=r^{-1}\left(R C_{r}\right)^{*},
$$

where, again, $C_{r}^{*}$ and $\left(R C_{r}\right)^{*}$ are the adjoint maps of $C_{r}$ and $R C_{r}$, respectively, with respect to the Euclidean scalar product. 
Remark 6.2 With the notations of (5.36), both in Proposition 5.3 and Theorem 5.1 we can replace the assumption $\alpha \in W^{3,2}\left(\Omega, E_{0}^{1}\right)$ by

$$
\alpha \in W^{2,2}\left(\Omega, E_{0}^{1}\right), \quad \delta_{g} d_{0}^{-1} d_{1} \alpha \in L^{2}(\Omega) \quad \text { and } \quad d_{2} d_{0}^{-1} d_{1} \alpha \in L^{2}\left(\Omega, \bigwedge^{2} \mathfrak{g}\right) .
$$

In particular, if $\mathbb{G}=\mathbb{H}^{1}, d_{0}^{-1} d_{1} \alpha$ is a 1 -form of weight 2 , and then $d_{2} d_{0}^{-1} d_{1} \alpha=0$, since there are no 2 -forms of weight 4 .

\section{Appendix: Carnot groups and Rumin's complex}

With the notations of Section 1 , let $(\mathbb{G}, \cdot)$ be a Carnot group of step $\kappa$ identified to $\mathbb{R}^{n}$ through exponential coordinates. As above, $\left\{e_{1}, \cdots, e_{n}\right\}$ is a basis of $\mathbb{R}^{n}$ adapted to the stratification of $\mathfrak{g}$. Moreover, let $X=\left\{X_{1}, \ldots, X_{n}\right\}$ be the family of left invariant vector fields such that $X_{i}(0)=e_{i}, i=1, \ldots, n$.

In the sequel, for undefined notations of multilinear algebra, we refer to Section 2 and to [21] and [12].

We recall the notion of weight of a form that is necessary to introduce the Rumin's complex.

Definition 7.1 If $\alpha \in \bigwedge^{1} \mathfrak{g}, \alpha \neq 0$, we say that $\alpha$ has pure weight $k$, and we write $w(\alpha)=k$, if $\alpha^{\natural} \in V_{k}$. More generally, if $\alpha \in \Lambda^{h} \mathfrak{g}$, we say that $\alpha$ has pure weight $k$ if $\alpha$ is a linear combination of covectors $\theta_{i_{1}} \wedge \cdots \wedge \theta_{i_{h}}$ with $w\left(\theta_{i_{1}}\right)+\cdots+w\left(\theta_{i_{h}}\right)=k$.

Remark 7.1 If $\alpha, \beta \in \Lambda^{h} \mathfrak{g}$ and $w(\alpha) \neq w(\beta)$, then $\langle\alpha, \beta\rangle=0$. Indeed, it is enough to notice that, if $w\left(\theta_{i_{1}} \wedge \cdots \wedge \theta_{i_{h}}\right) \neq w\left(\theta_{J_{1}} \wedge \cdots \wedge \theta_{J_{h}}\right)$, with $i_{1}<i_{2}<\cdots<i_{h}$ and $J_{1}<J_{2}<\cdots<J_{h}$, then for at least one of the indices $\ell=1, \ldots, h, i_{\ell} \neq J_{\ell}$, and hence $\left\langle\theta_{i_{1}} \wedge \cdots \wedge \theta_{i_{h}}, \theta_{J_{1}} \wedge \cdots \wedge \theta_{J_{h}}\right\rangle=0$.

We have ([3], formula (16))

$$
\bigwedge^{h} \mathfrak{g}=\bigoplus_{p=M_{h}^{\min }}^{M_{h}^{\max }} \bigwedge^{h, p} \mathfrak{g},
$$

where $\bigwedge^{h, p} \mathrm{~g}$ is the linear span of the $h$-covectors of weight $p$ and $M_{h}^{\min }, M_{h}^{\max }$ are respectively the smallest and the largest weight of left-invariant $h$-covectors.

Keeping in mind the decomposition (7.42), we can define in the same way several left invariant fiber bundles over $\mathbb{G}$, that we still denote with the same symbol $\bigwedge^{h, p} \mathfrak{g}$.

We notice also that the fiber $\bigwedge_{x}^{h} \mathrm{~g}$ (and hence the fiber $\bigwedge_{x}^{h, p} \mathrm{~g}$ ) can be endowed with a natural scalar product $\langle\cdot, \cdot\rangle_{x}$.

We denote by $\Omega^{h, p}$ the vector space of all smooth $h$-forms in $\mathbb{G}$ of pure weight $p$, i.e. the space of all smooth sections of $\bigwedge^{h, p} \mathfrak{g}$. We have

$$
\Omega^{h}=\bigoplus_{p=M_{h}^{\min }}^{M_{h}^{\max }} \Omega^{h, p} .
$$


The following crucial property of the weight follows from Cartan identity: see [32], Section 2.1:

Lemma 7.1 We have $d\left(\bigwedge^{h, p} \mathfrak{g}\right) \subset \bigwedge^{h+1, p} \mathfrak{g}$, i.e., if $\alpha \in \bigwedge^{h, p} \mathfrak{g}$ is a left invariant $h$-form of weight $p$ with $d \alpha \neq 0$, then $w(d \alpha)=w(\alpha)$.

Definition 7.2 (see, e.g., [3], [32]) Let now $\alpha=\sum_{\theta_{i}^{h} \in \Theta^{h, p}} \alpha_{i} \theta_{i}^{h} \in \Omega^{h, p}$ be a (say) smooth form of pure weight $p$. Then we can write

$$
d \alpha=d_{0} \alpha+d_{1} \alpha+\cdots+d_{\kappa} \alpha
$$

where $d_{0}$ does not increase the weight, $d_{1}$ increases the weight by 1 , and, more generally, $d_{i}$ increases the weight by $i$, when $i=0,1, \ldots, \kappa$. In particular, $d_{0}$ is an algebraic operator. We denote by $\delta_{0}$ its adjoint.

Lemma $7.2 d_{0}^{2}=0$, i.e. $\left(\Omega^{*}, d_{0}\right)$ is a complex.

Proof. Take $\alpha \in \Omega^{h, p}$, and write the identity $d^{2} \alpha=0$, gathering all terms according their weights. Since terms with different weights are orthogonal, this yields that all groups of given weight vanish. But the group of weight $p$ is precisely $d_{0}^{2} \alpha$, and we are done.

The following definition of intrinsic covectors (and therefore of intrinsic forms) is due to M. Rumin ([32], [30]).

Definition 7.3 If $0 \leq h \leq n$ we set

$$
E_{0}^{h}:=\operatorname{ker} d_{0} \cap \operatorname{ker} \delta_{0}=\operatorname{ker} d_{0} \cap\left(\operatorname{Im} d_{0}\right)^{\perp} \subset \Omega^{h}
$$

We refer to the elements of $E_{0}^{h}$ as to intrinsic $h$-forms on $\mathbb{G}$. Since the construction of $E_{0}^{h}$ is left invariant, this space of forms can be seen as the space of sections of a fiber subbundle of $\bigwedge^{h} \mathfrak{g}$, generated by left translation and still denoted by $E_{0}^{h}$. In particular $E_{0}^{h}$ inherits from $\wedge^{h} \mathrm{~g}$ the scalar product on the fibers.

Remark 7.2 There exists a left invariant orthonormal basis $\Xi_{0}^{h}=\left\{\xi_{j}\right\}$ of $E_{0}^{h}$ that is adapted to the filtration (7.42).

Since it is easy to see that $E_{0}^{1}=\operatorname{span}\left\{\theta_{1}, \ldots, \theta_{m}\right\}$, without loss of generality, we can take $\xi_{j}=\theta_{j}$ for $j=1, \ldots, m$.

Finally, we denote by $N_{h}^{\min }$ and $N_{h}^{\max }$ respectively the lowest and highest weight of forms in $E_{0}^{h}$.

We define now a (pseudo) inverse of $d_{0}$ as follows (see [3], Lemma 2.11):

Lemma 7.3 If $\beta \in \bigwedge^{h+1} \mathfrak{g}$, then there exists a unique $\alpha \in \bigwedge^{h} \mathfrak{g} \cap\left(\operatorname{ker} d_{0}\right)^{\perp}$ such that

$$
\delta_{0} d_{0} \alpha=\delta_{0} \beta . \quad \text { We set } \alpha:=d_{0}^{-1} \beta .
$$

In particular

$$
\alpha=d_{0}^{-1} \beta \quad \text { if and only if } \quad d_{0} \alpha-\beta \in \operatorname{ker} \delta_{0}=\mathcal{R}\left(d_{0}\right)^{\perp} .
$$

In addition, $d_{0}^{-1}$ preserves the weights. 
The following theorem summarizes the construction of the intrinsic differential $d_{c}$ (for details, see [32] and [3], Section 2) .

Theorem 7.1 The de Rham complex $\left(\Omega^{*}, d\right)$ splits in the direct sum of two sub-complexes $\left(E^{*}, d\right)$ and $\left(F^{*}, d\right)$, with

$$
E:=\operatorname{ker} d_{0}^{-1} \cap \operatorname{ker}\left(d_{0}^{-1} d\right) \quad \text { and } \quad F:=\mathcal{R}\left(d_{0}^{-1}\right)+\mathcal{R}\left(d d_{0}^{-1}\right) .
$$

We have

i) Let $\Pi_{E}$ be the projection on $E$ along $F$ (that is not an orthogonal projection). Then for any $\alpha \in E_{0}^{h, p}$, if we denote by $\left(\Pi_{E} \alpha\right)_{j}$ the component of $\Pi_{E} \alpha$ of weight $j$, then

$$
\begin{aligned}
\left(\Pi_{E} \alpha\right)_{p} & =\alpha \\
\left(\Pi_{E} \alpha\right)_{p+k+1} & =-d_{0}^{-1}\left(\sum_{1 \leq \ell \leq k+1} d_{\ell}\left(\Pi_{E} \alpha\right)_{p+k+1-\ell}\right) .
\end{aligned}
$$

Notice that $\alpha \rightarrow\left(\Pi_{E} \alpha\right)_{p+k+1}$ is an homogeneous differential operator of order $k+1$ in the horizontal derivatives.

ii) $\Pi_{E}$ is a chain map, i.e.

$$
d \Pi_{E}=\Pi_{E} d .
$$

iii) Let $\Pi_{E_{0}}$ be the orthogonal projection from $\Omega^{*}$ on $E_{0}^{*}$, then

$$
\Pi_{E_{0}}=I d-d_{0}^{-1} d_{0}-d_{0} d_{0}^{-1}, \quad \Pi_{E_{0}^{\perp}}=d_{0}^{-1} d_{0}+d_{0} d_{0}^{-1} .
$$

Notice that, since $d_{0}$ and $d_{0}^{-1}$ are algebraic, then formulas (7.44) hold also for covectors.

iv) $\Pi_{E_{0}} \Pi_{E} \Pi_{E_{0}}=\Pi_{E_{0}}$ and $\Pi_{E} \Pi_{E_{0}} \Pi_{E}=\Pi_{E}$.

Set now

$$
d_{c}=\Pi_{E_{0}} d \Pi_{E}: E_{0}^{h} \rightarrow E_{0}^{h+1}, \quad h=0, \ldots, n-1 .
$$

We have:

v) $d_{c}^{2}=0$;

vi) the complex $E_{0}:=\left(E_{0}^{*}, d_{c}\right)$ is exact;

vii) with respect to the bases $\Xi^{*}$, the intrinsic differential $d_{c}$ can be seen as a matrixvalued operator such that, if $\alpha$ has weight $p$, then the component of weight $q$ of $d_{c} \alpha$ is given by an homogeneous differential operator in the horizontal derivatives of order $q-p \geq 1$, acting on the components of $\alpha$.

Remark 7.3 We have also $E=\operatorname{ker} \delta_{0}+\operatorname{ker}\left(\delta_{0} d\right)$ and $F=\operatorname{Im} \delta_{0}+\operatorname{Im}\left(d \delta_{0}\right)$.

Proposition 7.1 Denote by $\delta_{c}=d_{c}^{*}$ the formal adjoint of $d_{c}$ in $L^{2}\left(\mathbb{G}, E_{0}^{*}\right)$.

Then assertions (3.13) still hold if we replace $d$ and $\delta$ by $d_{c}$ and $\delta_{c}$, respectively, or by $d_{0}, \delta_{0}$, respectively. 
Lemma 7.4 If $\omega \in E^{h}$ and $t(\omega)=0$, then $\omega \in * F^{\perp}$ (with respect to the $L^{2}$-product).

Proof. By [33], identity (2.27), $n(* \omega)=0$. First of all, we can show that

$$
* \omega \in \operatorname{ker} d_{0} \cap \operatorname{ker}\left(d_{0} \delta\right) .
$$

Indeed, by Proposition 7.1, $d_{0}(* \omega)=(-1)^{h} * \delta_{0} \omega=0$ and $d_{0} \delta * \omega=(-1)^{h+1} d_{0} * d \omega=$ $* \delta_{0} d \omega=0$. Take now $\alpha=\delta_{0} \xi+d \delta_{0} \eta \in F$. Clearly

$$
\int_{\mathcal{U}}\left\langle * \omega, \delta_{0} \xi\right\rangle d V=\int_{\mathcal{U}}\left\langle d_{0} * \omega, \xi\right\rangle d V=0 .
$$

On the other hand, by classical Green's formula

$$
\begin{aligned}
\int_{\mathcal{U}} & \left\langle\omega, d \delta_{0} \eta\right\rangle d V \\
& =\int_{\mathcal{U}}\left\langle\delta * \omega, \delta_{0} \eta\right\rangle d V+\int_{\partial \mathcal{U}} t\left(\delta_{0} \eta\right) \wedge * n(* \omega) \\
& \left.=\int_{\mathcal{U}}\left\langle\delta * \omega, \delta_{0} \eta\right\rangle d V \quad \text { (since } n(* \omega)=* t(\omega)\right) \\
& =\int_{\mathcal{U}}\left\langle d_{0} \delta * \omega, \eta\right\rangle d V=0 .
\end{aligned}
$$

This proves that $* \omega \in F^{\perp}$, and then the assertion follows.

Finally, we recall the definition of free Carnot group (see, for instance [6], Section 14.1).

Definition 7.4 Let $m \geq 2$ and $\kappa \geq 1$ be fixed integers. We say that $\mathfrak{f}_{m, \kappa}$ is the free Lie algebra with $m$ generators $x_{1}, \ldots, x_{m}$ and nilpotent of step $\kappa$ if:

i) $\mathfrak{f}_{m, k}$ is a Lie algebra generated by its elements $x_{1}, \ldots, x_{m}$, i.e. $\mathfrak{f}_{m, k}=\operatorname{Lie}\left(x_{1}, \ldots, x_{m}\right)$;

ii) $\mathfrak{f}_{m, \kappa}$ is nilpotent of step $\kappa$;

iii) for every Lie algebra $n$ nilpotent of step $\kappa$ and for every map $\phi$ from the set $\left\{x_{1}, \ldots, x_{m}\right\}$ to $\mathfrak{n}$, there exists a (unique) homomorphism of Lie algebras $\Phi$ from $\mathfrak{f}_{m, \kappa}$ to $\mathfrak{n}$ which extends $\phi$.

The Carnot group $\mathbb{G}$ is said free if its Lie algebra $g$ is isomorphic to a free Lie algebra.

When $\mathbb{G}$ is a free group, we can assume $\left\{X_{1}, \ldots, X_{n}\right\}$ a Grayson-Grossman-Hall basis of $\mathfrak{g}$ (see [22], [6], Theorem 14.1.10). This makes several computations much simpler. In particular, $\left\{\left[X_{i}, X_{j}\right], X_{i}, X_{j} \in V_{1}, i<j\right\}$ provides an orthonormal basis of $V_{2}$.

Theorem 7.2 ([19], Theorem 5.9) Let $\mathbb{G}$ be a free group of step $\kappa$. Then all forms in $E_{0}^{1}$ have weight 1 and all forms in $E_{0}^{2}$ have weight $\kappa+1$.

In particular, the differential $d_{c}: E_{0}^{1} \rightarrow E_{0}^{2}$ can be identified, with respect to the adapted bases $\Xi_{0}^{1}$ and $\Xi_{0}^{2}$, with a homogeneous matrix-valued differential operator of degree $\kappa$ in the horizontal derivatives.

Moreover, since $\Pi_{E_{0}}$ preserves the weights, if $\xi \in \bigwedge^{2, p} \mathfrak{g}$ with $p \neq \kappa+1$, then $\Pi_{E_{0}} \xi=0$. Indeed, $\Pi_{E_{0}} \xi$ has weight $p$, and therefore has to be zero, since $\Pi_{E_{0}} \xi \in \wedge^{2, \kappa+1} \mathfrak{g}$. 
Lemma 7.5 ([19], Lemma 6.3) If $\mathfrak{g}$ is a free algebra of step 2, then

1. $d_{0}\left(\bigwedge^{1} \mathfrak{g}\right)=\bigwedge^{2,2} \mathfrak{g}$

2. if $\theta_{i} \wedge \theta_{j} \in \wedge^{2,2} \mathfrak{g}$, then $d_{0}^{-1}\left(\theta_{i} \wedge \theta_{j}\right)=-\left[X_{i}, X_{j}\right]^{\natural}$;

3. if $\theta_{i} \wedge \theta_{j} \in \wedge^{2,2} \mathfrak{g}$, then $d_{0} d_{0}^{-1}\left(\theta_{i} \wedge \theta_{j}\right)=\theta_{i} \wedge \theta_{j}$;

4. if $\theta_{i} \wedge \theta_{j} \in \wedge^{2,3} \mathfrak{g}$ or $\theta_{i} \wedge \theta_{j} \in \bigwedge^{2,4} \mathfrak{g}$ then $d_{0}^{-1}\left(\theta_{i} \wedge \theta_{j}\right)=0$, so that again $d_{0}^{-1}\left(\theta_{i} \wedge \theta_{j}\right)=$ $-\left[X_{i}, X_{j}\right]^{\natural}$.

\section{References}

[1] K. Astala, T. Inaniec, and G. Martin, Elliptic partial differential equations and quasiconformal mappings in the plane, vol. 48 of Princeton Mathematical Series, Princeton University Press, Princeton, NJ, 2009.

[2] A. Baldi ANd B. Franchi, Differential forms in Carnot groups: a $\Gamma$-convergence approach, Calc. Var. Partial Differential Equations, 43 (2012), pp. 211-229.

[3] A. Baldi, B. Franchi, N. Tchou, and M. C. Tesi, Compensated compactness for differential forms in Carnot groups and applications, Adv. Math., 223 (2010), pp. 1555-1607.

[4] A. Baldi, B. Franchi, and M. C. Tesi, Compensated compactness in the contact complex of Heisenberg groups, Indiana Univ. Math. J., 57 (2008), pp. 133-186.

[5] - Differential forms, Maxwell equations and compensated compactness in Carnot groups, Lecture Notes of Seminario Interdisciplinare di Matematica, 7 (2008), pp. 21-40.

[6] A. Bonfiglioli, E. Lanconelli, and F. Uguzzoni, Stratified Lie groups and potential theory for their sub-Laplacians, Springer Monographs in Mathematics, Springer, Berlin, 2007.

[7] M. Born And E. Wolf, Principles of optics: Electromagnetic theory of propagation, interference and diffraction of light, Pergamon Press, New York-London-Paris-Los Angeles, 1959.

[8] G. Citti and M. Manfredini, Uniform estimates of the fundamental solution for a family of hypoelliptic operators, Potential Anal., 25 (2006), pp. 147-164.

[9] M. Costabel, A coercive bilinear form for Maxwell's equations, J. Math. Anal. Appl., 157 (1991), pp. 527-541.

[10] G. Dal Maso, An introduction to $\Gamma$-convergence, Progress in Nonlinear Differential Equations and their Applications, 8, Birkhäuser Boston Inc., Boston, MA, 1993.

[11] D. Danielli, N. Garofalo, and D.-M. Nhieu, Non-doubling Ahlfors measures, perimeter measures, and the characterization of the trace spaces of Sobolev functions in Carnot-Carathéodory spaces, Mem. Amer. Math. Soc., 182 (2006), pp. x+119.

[12] H. Federer, Geometric measure theory, Die Grundlehren der mathematischen Wissenschaften, Band 153, Springer-Verlag New York Inc., New York, 1969.

[13] G. B. Folland, Subelliptic estimates and function spaces on nilpotent Lie groups, Ark. Mat., 13 (1975), pp. 161-207.

[14] G. B. Folland and E. M. Stein, Hardy spaces on homogeneous groups, vol. 28 of Mathematical Notes, Princeton University Press, Princeton, N.J., 1982. 
[15] B. Franchi, V. Penso, and R. Serapioni, Remarks on Lipschitz domains in Carnot groups, in Geometric control and sub-Riemannian geometry, U. Boscain, J.-P. Gauthier, A. Sarychev, M. Sigalotti, and G. Stefani, eds., Springer INdAM Series, Springer, Nov. 2013, pp. 153-166.

[16] B. Franchi, R. Serapioni, and F. Serra Cassano, On the structure of finite perimeter sets in step 2 Carnot groups, J. Geom. Anal., 13 (2003), pp. 421-466.

[17] B. Franchi, N. Tchou, and M. C. Tesi, Div-curl type theorem, H-convergence and Stokes formula in the Heisenberg group, Commun. Contemp. Math., 8 (2006), pp. 67-99.

[18] B. Franchi and M. C. Tesi, Faraday's form and Maxwell's equations in the Heisenberg group, Milan J. Math., 77 (2009), pp. 245-270.

[19] _ Wave and Maxwell's equations in Carnot groups, Commun. Contemp. Math., 14 (2012), pp. 1250032, 62.

[20] Z. GE, Collapsing Riemannian metrics to Carnot-Carathéodory metrics and Laplacians to subLaplacians, Canad. J. Math., 45 (1993), pp. 537-553.

[21] M. Giaquinta, G. Modica, And J. Součex, Cartesian currents in the calculus of variations. I, vol. 37 of Ergebnisse der Mathematik und ihrer Grenzgebiete. 3. Folge. A Series of Modern Surveys in Mathematics [Results in Mathematics and Related Areas. 3rd Series. A Series of Modern Surveys in Mathematics], Springer-Verlag, Berlin, 1998.

[22] M. Grayson and R. Grossman, Models for free nilpotent Lie algebras, J. Algebra, 135 (1990), pp. 177-191.

[23] A. Isopoussu, K. Peltonen, And J. T. Tyson, Quasiregular maps and the conductivity equation in the Heisenberg group, in In the tradition of Ahlfors-Bers. VI, vol. 590 of Contemp. Math., Amer. Math. Soc., Providence, RI, 2013, pp. 61-75.

[24] C. E. Kenig, M. Salo, and G. Uhlmann, Inverse problems for the anisotropic Maxwell equations, Duke Math. J., 157 (2011), pp. 369-419.

[25] R. LeIs, Zur Theorie elektromagnetischer Schwingungen in anisotropen inhomogenen Medien, Math. Z., 106 (1968), pp. 213-224.

[26] G. Lu, Polynomials, higher order Sobolev extension theorems and interpolation inequalities on weighted Folland-Stein spaces on stratified groups, Acta Math. Sin. (Engl. Ser.), 16 (2000), pp. 405-444.

[27] R. Monti and D. Morbidelli, Trace theorems for vector fields, Math. Z., 239 (2002), pp. 747 776.

[28] L. P. Rothschild and E. M. Stein, Hypoelliptic differential operators and nilpotent groups, Acta Math., 137 (1976), pp. 247-320.

[29] M. Rumin, Formes différentielles sur les variétés de contact, J. Differential Geom., 39 (1994), pp. 281-330.

[30] - Differential geometry on C-C spaces and application to the Novikov-Shubin numbers of nilpotent Lie groups, C. R. Acad. Sci. Paris Sér. I Math., 329 (1999), pp. 985-990.

[31] - Sub-Riemannian limit of the differential form spectrum of contact manifolds, Geom. Funct. Anal., 10 (2000), pp. 407-452.

[32] _ - Around heat decay on forms and relations of nilpotent Lie groups, in Séminaire de Théorie Spectrale et Géométrie, Vol. 19, Année 2000-2001, vol. 19 of Sémin. Théor. Spectr. Géom., Univ. Grenoble I, Saint, 2001, pp. 123-164.

[33] G. Schwarz, Hodge decomposition — a method for solving boundary value problems, vol. 1607 of Lecture Notes in Mathematics, Springer-Verlag, Berlin, 1995. 
[34] B. Zwiebach, A first course in string theory, Cambridge University Press, Cambridge, second ed., 2009. With a foreword by David Gross. 